\title{
A new wavelet family based on second-order LTI-systems
}

\author{
Tariq Abuhamdia', Saied Taheri' and John Burns ${ }^{2}$
}

\begin{abstract}
In this paper, a new family of wavelets derived from the underdamped response of second-order Linear-Time-Invariant (LTI) systems is introduced. The most important criteria for a function or signal to be a wavelet is the ability to recover the original signal back from its continuous wavelet transform. We show that it is possible to recover back the original signal once the Second-Order Underdamped LTI (SOULTI) wavelet is applied to decompose the signal. It is found that the SOULTI wavelet transform of a signal satisfies a linear differential equation called the reconstructing differential equation, which is closely related to the differential equation that produces the wavelet. Moreover, a time-frequency resolution is defined based on two different approaches. The new transform has useful properties; a direct relation between the scale and the frequency, unique transform formulas that can be easily obtained for most elementary signals such as unit step, sinusoids, polynomials, and decaying harmonic signals, and linear relations between the wavelet transform of signals and the wavelet transform of their derivatives and integrals. The results obtained are presented with analytical and numerical examples. Signals with constant harmonics and signals with time-varying frequencies are analyzed, and their evolutionary spectrum is obtained. Contour mapping of the transform in the time-scale and the timefrequency domains clearly detects the change of the frequency content of the analyzed signals with respect to time. The results are compared with other wavelets results and with the short-time fourier analysis spectrograms. At the end, we propose the method of reverse wavelet transform to mitigate the edge effect.
\end{abstract}

\section{Keywords}

SOULTI-wavelets, second-order systems wavelets, LTI-wavelets, second-order linear-time-invariant wavelets, time-frequency analysis, spectrogram, scalogram, chirp analysis, frequency-identification, edge-effect, forward-wavelet-transform, forward wavelet transform, reverse-wavelet-transform, reverse wavelet transform

\section{Introduction}

Wavelets provide a powerful tool to analyze signals and extract information from them. They are capable of extracting frequency, time, and nonharmonic information. These potentials lured many scholars to use them in the analysis of dynamic systems. Scholars have used wavelets for system identification, system modeling, system response solution, and even control design. For broad and extensive survey on the use of wavelets in systems and control, the reader is referred to Abuhamdia and Taheri (2015).

Mathematically, the wavelet transform is an inner product between a function and a set of basis functions which are all derived from a single function called the mother wavelet. It measures how much parallelism exists between the analyzed function and the set of basis functions. Therefore, if we seek to extract some features from a signal, then the analyzing wavelet family should also have these features. This is similar to the way we measure the periodicity of a signal by making inner product with the harmonic functions because of their periodicity.

This idea also implies that if we want to use timefrequency analysis on a dynamic system by analyzing its response, a better understanding can be developed if

\footnotetext{
'Center for Tire Research (CenTire), Virginia Polytechnic Institute and State University, USA

${ }^{2}$ Interdisciplinary Center for Applied Mathematics (ICAM), Virginia

Polytechnic Institute and State University, USA

Received: 18 May 2016; accepted: 4 September 2016

\section{Corresponding author:}

Tariq Abuhamdia, Department of Mechanical Engineering, Virginia Tech, I00T Randolph Hall, 460 Old Turner St, Blacksburg, VA, 24060, USA.

Email: atariqm@vt.edu
} 
the analyzing wavelet is close in characteristics to systems responses and behaviors.

This observation led to investigating the characteristics of the underdamped second-order response of Linear Time-Invariant (LTI) systems to find if it can serve as a mother wavelet. The underdamped secondorder impulse response of LTI systems is oscillatory and decaying exponentially, and it dies out to effective zero well within the defined period. Furthermore, its frequency domain representation is a second-order filter that can effectively extract certain frequency bands from the signals.

It was intuitive to try to construct families of wavelets from the building blocks of systems responses, especially LTI systems. Such families of wavelets could be useful in systems characterization and provide new perspective for understanding systems and how their responses evolve. It was a remarkable coincidence that Robinson (1962) called the response of a secondorder LTI systems a wavelet. However, the closest point in this track was using the response of secondorder LTI-systems as pseudo wavelets (Freudinger et al., 1998; Hou and Hera, 2001). They were considered pseudo wavelets because they failed to satisfy the reconstruction conditions, namely the inverse wavelet transform was not possible. In addition to those efforts, Newland (1993) proposed the harmonic wavelets which possess the important advantages of being orthogonal and having excellent frequency localization. Moreover, they can be viewed as perfect band-pass filters. Jezequel and Argoul (1986) used a transfer function in the frequency domain (ratio of zeros and poles) as a kernel for an integral transform that transforms signals from the frequency domain to another two-dimensional domain whose axes represent some parameters of the model represented by kernel.

The response of second-order systems had been used before to analyze signals for different purposes and under different names but as a pseudo wavelet or dictionary of wavelets. Freudinger et al. (1998) defined the Laplace wavelet, by

$$
\psi(f, \zeta, \tau, t)=\left\{\begin{array}{lc}
A e^{\frac{-\zeta}{\sqrt{1-\zeta^{2}}} 2 \pi \vartheta(t-\tau)} e^{-j 2 \pi \vartheta(t-\tau)} & t \in\left[\tau, \tau+T_{s}\right] \\
0 & \text { else }
\end{array}\right.
$$

where $\zeta$ represents the damping ratio, $\vartheta$ is the frequency, and $T_{s}$ is the effective duration of the wavelet that defines the effective compact support. They formed a dictionary of wavelets but not a basis or frame. Hou and Hera (2001) used the the magnitude of secondorder LTI systems response in the frequency domain as a pseudo wavelet and defined it by

$$
\Psi\left(\omega, \omega_{0}, \zeta_{0}\right)= \begin{cases}\rho \frac{\omega_{0}^{2}}{\sqrt{\left(\omega^{2}-\omega_{0}^{2}\right)^{2}+\left(2 \zeta_{0} \omega_{0} \omega\right)^{2}}} & \omega \geq 0 \\ 0 & \omega<0\end{cases}
$$

and used the continuous wavelet transform but in the frequency domain to identify the parameters of dynamic systems by mapping the match between the system frequency response and the pseudo wavelet.

In the following section, we show that it is possible to construct a family of wavelets from the response of Second-Order Underdamped LTI (SOULTI) systems that we call, for brevity, the SOULTI wavelets. We show that their inverse continuous wavelet transform exists and define the basic properties an analyst needs to perform time-scale or time-frequency analysis. In Section 2, we define the SOULTI wavelet families. Section 3 constructs and proves the existence of the inverse wavelet transform for the SOULTI wavelets. Section 4 explores the basic properties of the SOULTI wavelet and the associated transform and lists the SOULTI wavelet transform for elementary signals. Section 5 defines the time and the frequency properties of the wavelet and derives different definitions for the time-frequency resolution of the wavelet transform. Section 6 presents an application with numerical examples for analyzing signals with different frequency characteristics, and Section 7 addresses the edge effect and proposes a solution to reduce its influence on the analysis.

\section{Second-order underdamped LTI wavelets}

Second-order LTI systems are very common in most dynamic fields of science. The mechanical mass-springdamper system, shown in Figure 1(a), and the RLC-electrical circuit, shown in Figure 1(b), are typical examples of such systems. The response of the SOULTI system in Figure 1, for the impulse input $\omega_{n}^{2} \delta(t)$ is given by

$$
h(t)=\left(\frac{\omega_{n}}{\sqrt{1-\zeta^{2}}}\right) e^{-\zeta \omega_{n} t} \sin \left(\omega_{n} \sqrt{1-\zeta^{2}} t\right) u(t)
$$

where $\zeta<1$ is the damping ratio, $\omega_{n}$ is the natural frequency and $u(t)$ is the Heaviside step function. The impulse input is scaled by $\omega_{n}^{2}$ to simplify the derivation of the frequency properties in Sections 4 and 5 . The damped frequency $\omega_{d}$ of the underdamped system is given by

$$
\omega_{d}=\omega_{n} \sqrt{1-\zeta^{2}}
$$




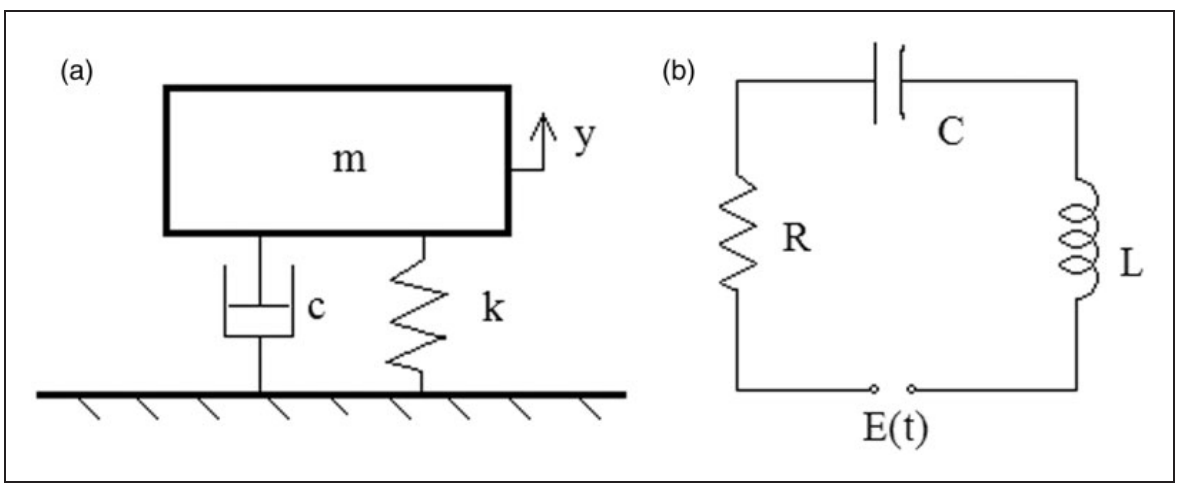

Figure I. (a) Mass-spring-damper system; (b) RLC electrical circuit.

so equation (3) can be rewritten in terms of $\omega_{d}$ as

$$
h(t)=\left(\frac{\omega_{d}}{1-\zeta^{2}}\right) e^{-\zeta \frac{\omega_{d}}{\sqrt{1-\zeta^{2}} t} \sin \left(\omega_{d} t\right) u(t)}
$$

Let the reciprocal of the damped frequency, defined in equation (4), be the scaling parameter as

$$
s=\frac{1}{\omega_{d}}
$$

Substitute equation (6) into equation (5) to get

$$
h(t)=\left(\frac{s^{-1}}{1-\zeta^{2}}\right) e^{-\zeta \frac{\frac{t}{s}}{\sqrt{1-\zeta^{2}}}} \sin \left(\frac{t}{s}\right) u(t)
$$

which is the impulse response in terms of the scaling parameter $s$ where $s \in(0, \infty)$. Now, the SOULTI wavelet can be defined as

$\psi_{\zeta}^{s, \tau}=\psi_{\zeta}\left(\frac{t-\tau}{s}\right)=\left(\frac{s^{-p}}{1-\zeta^{2}}\right) e^{\frac{-\zeta}{\sqrt{1-\zeta^{2}}}\left(\frac{t-\tau}{s}\right)} \sin \left(\frac{t-\tau}{s}\right) u(t-\tau)$

which represents the real part of the Laplace wavelet defined by Freudinger et al. (1998). The parameter $p$ is used to give the wavelet a preservation property. For example, when the wavelet is scaled, its energy content is also scaled, so to preserve the energy of the $L^{2}$ norm under scaling we use $p=1 / 2$. However, to preserve the $L^{1}$ norm of the wavelet, namely

$$
\int_{-\infty}^{\infty}\left|\psi_{\zeta}\left(\frac{t}{s}\right)\right| \mathrm{d} t=\int_{-\infty}^{\infty}\left|\psi_{\zeta}(t)\right| \mathrm{d} t
$$

we use $\mathrm{p}=1$. Figure 2 graphs the SOULTI wavelet versus time showing its time properties. The wavelet function defined in equation (8) represents more than one family of wavelets, where each family is linked to a single value of $\zeta$, where $0<\zeta<1$. It retains the LTI second-order response characteristics completely.
Suppose that $J \subset(a, \infty) \subset \mathbb{R}$, and let $f(t): J \rightarrow \mathbb{R}$ and $f(t) \in L^{1}$ and is exponentially bounded, see section (2.1). The SOULTI wavelet transform of $f(t)$ can be now defined by the generic continuous wavelet transform definition

$$
\mathcal{W}_{\zeta}\{f(t)\}=\tilde{f}_{\zeta}(\tau, s)=\int_{-\infty}^{\infty} f(t) \psi_{\zeta}\left(\frac{t-\tau}{s}\right) \mathrm{d} t, \quad \tau \in(-\infty, \infty)
$$

The SOULTI wavelet transform in equation (10) offers a measurement of similarity between any signal and the response of second-order LTI systems for characterization and identification purposes. In addition, The SOULTI wavelet gives a direct and simple relationship between scale and frequency as shown in equation (6), where the frequency is the reciprocal of the scale.

\section{I. Region of convergence}

The region of convergence of the SOULTI transform defines the region $\Gamma \subset S \times T$, where $s \in S=$ $(0, \infty), \tau \in T=(-\infty, \infty)$, in which the transform in equation (10) converges to a finite value. Before exploring such region, notice that $f(t)$ has to be exponentially bounded in order for the transform in equation (10) to converge.

Exponential boundedness is defined in the following; define $J \subset(a, \infty) \subset \mathbb{R}$, and let $f(t): J \rightarrow \mathbb{R}$, if $\exists \alpha, k \in \mathbb{R}$ such that $|f(t)| \leq\left|k e^{\alpha t}\right| \forall t \in J$, then $f(t)$ is exponentially bounded.

If $f(t)$ is exponentially bounded, then the SOULTI transform is convergent in the scale region defined by

$$
0<s<\frac{\zeta}{\alpha \sqrt{1-\zeta^{2}}}
$$

When the time domain is considered for convergence, i.e. considering the values of the time shift $\tau$ that renders the transform convergent, we have to be careful about the uniqueness of the transform because 


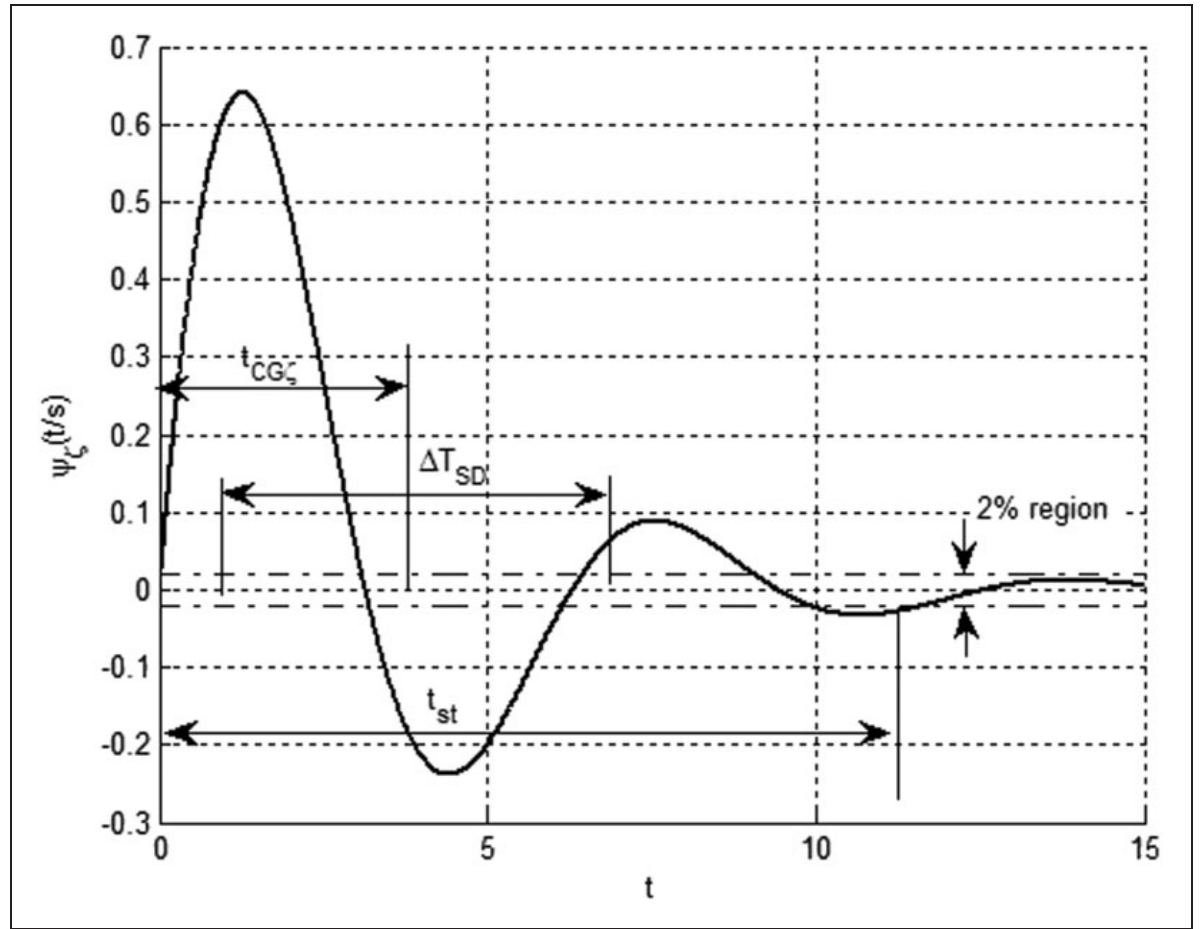

Figure 2. The SOULTI mother wavelet time function at $\zeta=0.3, s=I$, and $p=I$.

the transform on finite time interval could be identical for two different functions on a set of measure greater than zero. Therefore, the time region has to be explicitly indicated on the transform, and uniqueness is not achieved in this case. In the next section, we will define the inverse continuous wavelet transform with respect to the SOULTI wavelet.

\section{SOULTI wavelet inverse transform}

Notice that equation (10) represents an inner product in the time domain, which is equivalent to the inner product in the frequency domain according to Plancherel's theorem (Yoshida, 1965). Applying Plancherel's theorem to equation (10) yields

$$
\tilde{f}_{\zeta}(\tau, s)=\left\langle f(t), \psi_{\zeta}^{\tau, s}\right\rangle=\frac{1}{2 \pi}\left\langle F(\omega), \Psi_{\zeta}^{\tau, s}(\omega)\right\rangle
$$

where $\Psi_{\zeta}^{\tau, s}(\omega)$ is the Fourier transform of $\psi_{\zeta}^{\tau, s}$, and using the Fourier transform shift and scale properties it can be expressed in terms of the Fourier transform of the mother wavelet, $\Psi_{\zeta}(\omega)$, as

$$
\mathcal{F}\left\{\psi_{\zeta}^{\tau, s}\right\}=s e^{-j \omega \tau} \Psi_{\zeta}(s \omega)
$$

Using Plancherels theorem, equation (10) becomes

$$
\tilde{f}_{\zeta}(\tau, s)=\frac{s}{2 \pi} \int_{-\infty}^{\infty} e^{j \omega \tau} \overline{\Psi_{\zeta}(s \omega)} F(\omega) \mathrm{d} \omega
$$

where the conjugate of $s e^{-j \omega \tau} \Psi_{\zeta}(s \omega)$ is substituted in the inner product. Note that the integral in the right side of equation (14) represents the inverse Fourier transform of $\overline{\Psi_{\zeta}(s \omega)} F(\omega)$. Applying the Fourier transform to equation (14) yields

$$
\int_{-\infty}^{\infty} e^{-j \omega \tau} \tilde{f}_{\zeta}(\tau, s) \mathrm{d} \tau=\frac{s}{2 \pi} \overline{\Psi_{\zeta}(s \omega)} F(\omega)
$$

In general, we cannot divide both sides by $s \overline{\Psi_{\zeta}(s \omega)}$ because it could vanish at some values of $\omega$ or $s$. However, in our case $s \overline{\Psi_{\zeta}(s \omega)}$ is given by

$$
s \overline{\Psi_{\zeta}(s \omega)}=\frac{\frac{s^{1-p}}{1-\zeta^{2}}}{-\omega^{2} s^{2}+\frac{1}{1-\zeta^{2}}-j \frac{2 \zeta \omega s}{\sqrt{1-\zeta^{2}}}}
$$

which does not vanish for any value of $\omega$ or $s \in(0, \infty)$.

Figure 3 shows the wavelet spectrum magnitude, which is equivalent to its conjugate spectrum 


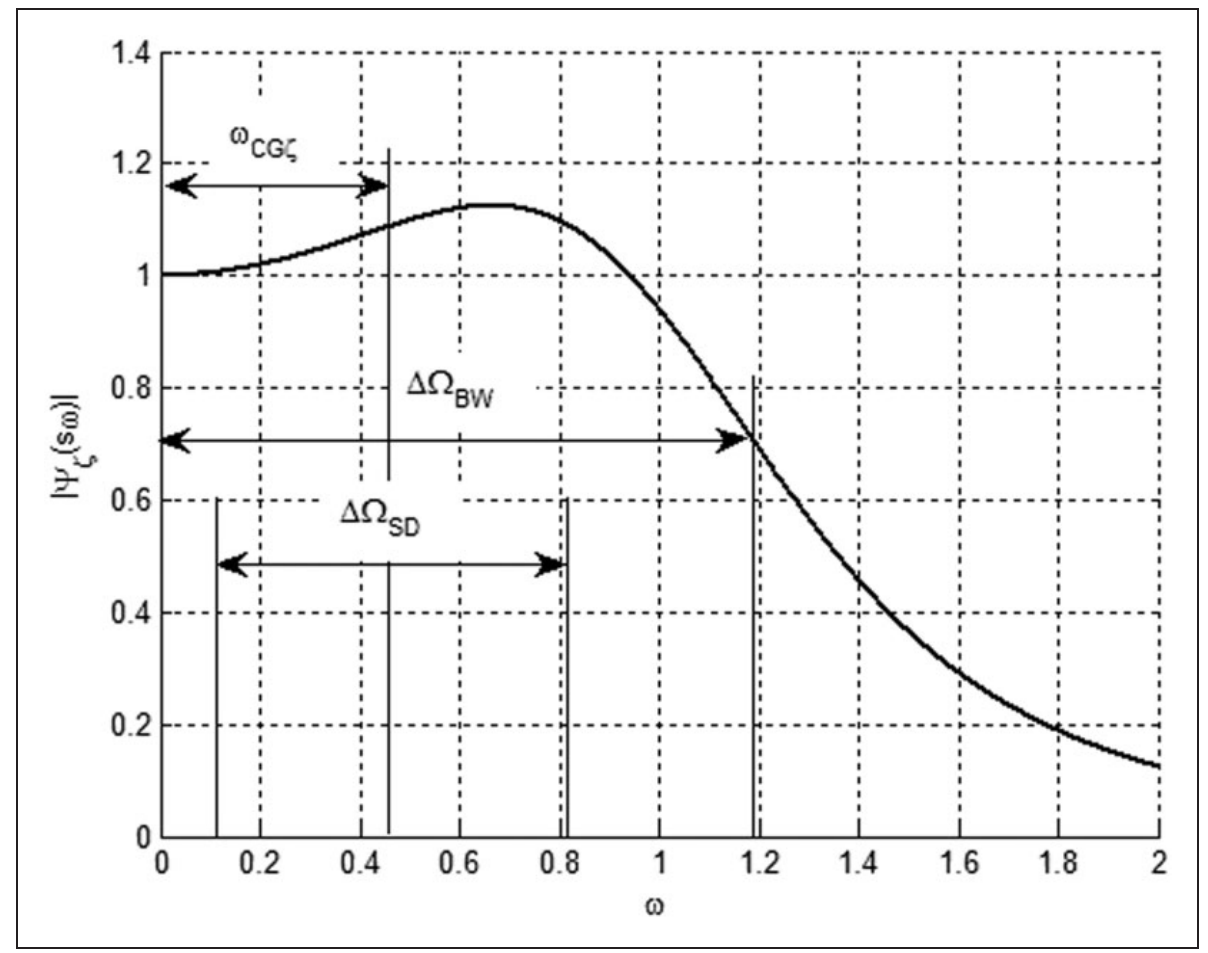

Figure 3. SOULTI wavelet in the frequency domain with the mean frequency $\omega_{C G \zeta}$, the standard deviation-based frequency window $\Delta \Omega_{S D}$, and the (half-power)-based frequency window $\Delta \Omega_{B W} \zeta=0.5, s=1$, and $p=1$.

magnitude. The curve never crosses the zero axis and decays asymptotically to zero. Consequently dividing by $s \overline{\Psi_{\zeta}(s \omega)}$ is a legitimate operation. Therefore, we can solve for $F(\omega)$ in equation (15) to get

$$
F(\omega)=\frac{1}{s \overline{\Psi_{\zeta}(s \omega)}} \int_{-\infty}^{\infty} e^{-j \omega \tau} \tilde{f}_{\zeta}(\tau, s) \mathrm{d} \tau
$$

To retrieve $f(t)$, take the inverse Fourier transform of equation (17), so the inverse wavelet transform with respect to the SOULTI wavelet becomes

$\mathcal{W}_{\zeta}^{-1}\left\{\tilde{f}_{\zeta}(\tau, s)\right\}=f(t)=\int_{-\infty}^{\infty} \int_{-\infty}^{\infty} \frac{e^{j \omega t}}{s \overline{\Psi_{\zeta}(s \omega)}} e^{-j \omega \tau} \tilde{f}_{\zeta}(\tau, s) \mathrm{d} \tau \mathrm{d} \omega$

Equation (18) forms the inverse wavelet transform with respect to the SOULTI wavelet or the reconstruction formula of the original wavelet definition shown in equation (10). If $f(t)$ is differentiable, we can use a simpler and probably more practical inverse formula to retrieve $f(t)$ back from its wavelet transform.

Theorem 1. Let $J \subset(a, \infty) \subset \mathbb{R}$, and let $f(t): J \rightarrow \mathbb{R}$ be differentiable and exponentially bounded, and let the SOULTI wavelet transform of $f(t)$ be given by equation (10), then the inverse wavelet transform satisfies the identity

$$
\begin{aligned}
f(t)= & \mathcal{W}_{\zeta}^{-1}\left\{\tilde{f}_{\zeta}(t, s)\right\}=s^{p-1}\left[\left(1-\zeta^{2}\right) s^{2} \frac{d^{2}\left(\tilde{f}_{\zeta}(t, s)\right)}{d t^{2}}\right. \\
& \left.-2 \sqrt{1-\zeta^{2}} \zeta s \frac{\mathrm{d}\left(\tilde{f}_{\zeta}(t, s)\right)}{d t}+\tilde{f}_{\zeta}(t, s)\right]
\end{aligned}
$$

Proof. Substituting $s \overline{\Psi_{\zeta}(s \omega)}$ from equation (16) into equation (18) gives

$$
\begin{aligned}
f(t)= & \int_{-\infty}^{\infty} \frac{e^{j \omega t}\left(1-\zeta^{2}\right)}{s^{1-p}}\left(-\omega^{2} s^{2}+\frac{1}{1-\zeta^{2}}-j \frac{2 \zeta \omega s}{\sqrt{1-\zeta^{2}}}\right) \\
& \times \int_{-\infty}^{\infty} e^{-j \omega t} \tilde{f}_{\zeta}(t, s) \mathrm{d} t \mathrm{~d} \omega
\end{aligned}
$$

Note that $\tau$ is substituted by $t$ inside the second integral. Using the operator notation for the Fourier transform, equation (20) becomes

$$
f(t)=s^{p-1} \mathcal{F}^{-1}\left\{\left(-\omega^{2} s^{2}\left(1-\zeta^{2}\right)+1-j \sqrt{1-\zeta^{2}} 2 \zeta \omega s\right) \mathcal{F}\left\{\tilde{f}_{\zeta}(t, s)\right\}\right\}
$$


Applying the linear operators properties (Naylor and Sell, 2000) to equation (21) gives

$$
\begin{aligned}
f(t)= & s^{p-1} \mathcal{F}^{-1}\left\{-\omega^{2} s^{2}\left(1-\zeta^{2}\right) \mathcal{F}\left\{\tilde{f}_{\zeta}(t, s)\right\}\right\} \\
& +s^{p-1} \mathcal{F}^{-1}\left\{\mathcal{F}\left\{\tilde{f}_{\zeta}(t, s)\right\}\right\} \\
& -s^{p-1} \mathcal{F}^{-1}\left\{j \sqrt{1-\zeta^{2}} 2 \zeta \omega s \mathcal{F}\left\{\tilde{f}_{\zeta}(t, s)\right\}\right\}
\end{aligned}
$$

and after applying the Fourier transform differentiation property to equation (22) we arrive at equation (19).

This provides a simple and direct method in the time domain to calculate the inverse wavelet transform with respect to the SOULTI wavelet. However, in order for the formula in equation (19) to apply, $\tilde{f}_{\zeta}(\tau, s)$ has to be at least twice differentiable with respect to time. When considering the transform that defines $\tilde{f}_{\zeta}(\tau, s)$ in equation $(10)$, we find that $\tilde{f}_{\zeta}(\tau, s)$ is twice differentiable with respect to time if $f(t)$ is differentiable. So if $f(t)$ is exponentially bounded and $f(t) \in C^{1}$, then its SOULTI wavelet transform is unique and $f(t)$ can be retrieved using equation (19).

Equation (19) also provides information about the uniqueness of the SOULTI wavelet transform. The inverse wavelet transform given by equation (19) is a linear second-order differential equation, which we will call the Reconstructing Differential Equation. The original function $f(t)$ is the input function and its wavelet transform at scale $s$ is a solution or part of the response. However, the other conditions must be satisfied in order for equation (19) to server as inverse formula for the SOULTI wavelet transform.

Corollary 2. Let $J \subset(a, \infty) \subset \mathbb{R}$, and let $f(t): J \rightarrow \mathbb{R}$ be differentiable and exponentially bounded, then the
SOULTI wavelet transform with respect to the wavelet family $\psi_{\zeta}\left(\frac{t-\tau}{s}\right)$ of $f(t)$ at scale $s$ is a solution of the following nonhomogeneous differential equation

$$
f(t)=s^{p-1}\left[\left(1-\zeta^{2}\right) s^{2} \frac{d^{2}(y(t))}{d t^{2}}-2 \sqrt{1-\zeta^{2}} \zeta s \frac{d(y(t))}{d t}+y(t)\right]
$$

Proof. The proof follows by direct substitution. Fix $s$, so it can be treated as a constant. Now, suppose that a solution of equation (23) is given by

$$
y_{p}(t)=\tilde{f}_{\zeta}(t, s)
$$

Substitute $y_{p}(t)$ back into the right side of equation (23) to get

$$
\begin{aligned}
G(t, s)= & s^{p-1}\left[\left(1-\zeta^{2}\right) s^{2} \frac{d^{2}}{d t^{2}}\left(\tilde{f}_{\zeta}(t, s)\right)\right. \\
& \left.-2 \sqrt{1-\zeta^{2}} \zeta s \frac{d}{d t}\left(\tilde{f}_{\zeta}(t, s)\right)+\tilde{f}_{\zeta}(t, s)\right]
\end{aligned}
$$

but we just proved by Theorem 1 that $G(t, s)=f(t)$.

\section{SOULTI transform of elementary signals and its properties}

Let us examine the validity of equation (19) with an example. Let $f(t)=e^{-\beta t}$, then its SOULTI wavelet transform is given by

$$
\tilde{f}_{\zeta}(\tau, s)=\frac{s^{-p}}{1-\zeta^{2}} \int_{-\tau}^{\infty} e^{-\beta t} e^{\frac{-\zeta}{\sqrt{1-\zeta^{2}}}\left(\frac{t-\tau}{s}\right)} \sin \left(\frac{t-\tau}{s}\right) \mathrm{d} t
$$

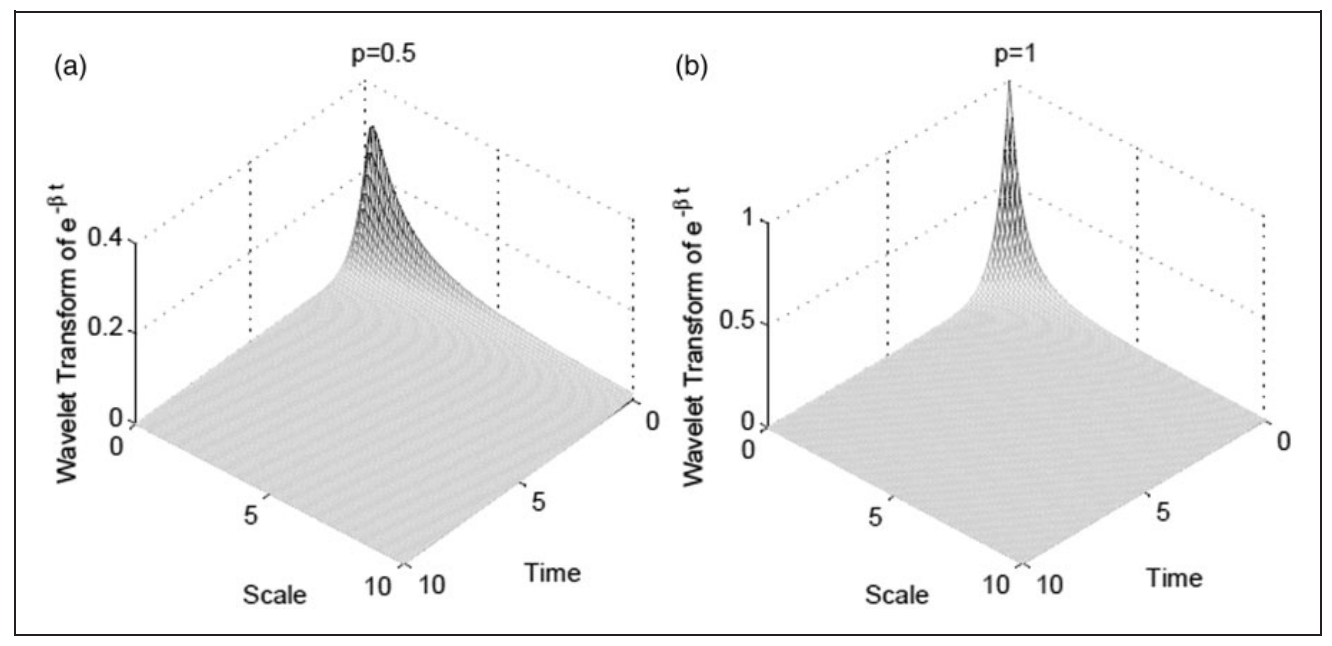

Figure 4. SOULTI Wavelet transform surface of the decaying exponential function at $\zeta=0.7$ and $\beta=2$. 
which can be evaluated using the integration by parts technique to give

$$
\tilde{f}_{\zeta}(\tau, s)=e^{-\beta \tau} \frac{s^{1-p}}{\left(1-\zeta^{2}\right) \beta s^{2}+2 \sqrt{1-\zeta^{2}} \zeta \beta s+1}
$$

which represents an analytical formula in terms of the scale $s$, the time shift $\tau$, and the wavelet damping ratio $\zeta$, in addition to the decay rate $\beta$.

Note that the transform of $e^{-\beta t}$ in equation (27) consists of a multiplication of two functions, a function of time and a function of scale. Note also, that the transform is very similar to the Laplace Transform of a delayed and scaled function. Figure 4 shows the wavelet transform of the exponential function as described in equation (27).

Let us now evaluate the SOULTI inverse transform by using the formula in equation (19). Differentiating equation (27) with respect to time twice and substituting the result into the right hand side of equation (27) and substituting $\tau$ by $t$ yields

$$
\begin{aligned}
& s^{p-1}\left[\left(\left(1-\zeta^{2}\right) s^{2} \beta^{2} e^{-\beta t}+e^{-\beta t}+2 \sqrt{1-\zeta^{2}} \zeta \beta s e^{-\beta t}\right)\right. \\
& \left.\quad \times\left(\frac{s^{1-p}}{\left(1-\zeta^{2}\right) \beta s^{2}+2 \sqrt{1-\zeta^{2}} \zeta \beta s+1}\right)\right]=e^{-\beta t} \Im \boldsymbol{\square}
\end{aligned}
$$

We can use the result in equation (27) to find the SOULTI wavelet transform for the sinusoidal functions. Table 1 lists the SOULTI wavelet transform for some elementary signals. Figure 5 shows the wavelet transform scalogram of the $\cos (\omega t)$ function.

Lemma 3. Let $J \subset(a, \infty) \subset \mathbb{R}$, and let $x(t): J \rightarrow \mathbb{R}$ be differentiable and exponentially bounded as defined in Theorem 1, and the SOULTI wavelet transform of $x(t)$ be given by equation (10), then the SOULTI wavelet transform of $\dot{x}(t)$ is given by

$$
\begin{aligned}
\tilde{\dot{x}}_{\zeta}(\tau, s) & =\frac{e^{\frac{-\zeta}{\sqrt{1-\zeta^{2}}} \theta}}{s \sqrt{1-\zeta^{2}}} \tilde{x}_{\zeta}(\tau+s \theta, s) \\
\theta & =\tan ^{-1}\left(\frac{\sqrt{1-\zeta^{2}}}{\zeta}\right)
\end{aligned}
$$

Proof. Substitute $\dot{x}(t)$ in equation (10) to have

$$
\tilde{\dot{x}}_{\zeta}(\tau, s)=\int_{\tau}^{\infty} \dot{x}(t) \psi_{\zeta}\left(\frac{t-\tau}{s}\right) \mathrm{d} t, \quad \tau \in(-\infty, \infty)
$$

Table I. SOULTI wavelet transform for basic signals.

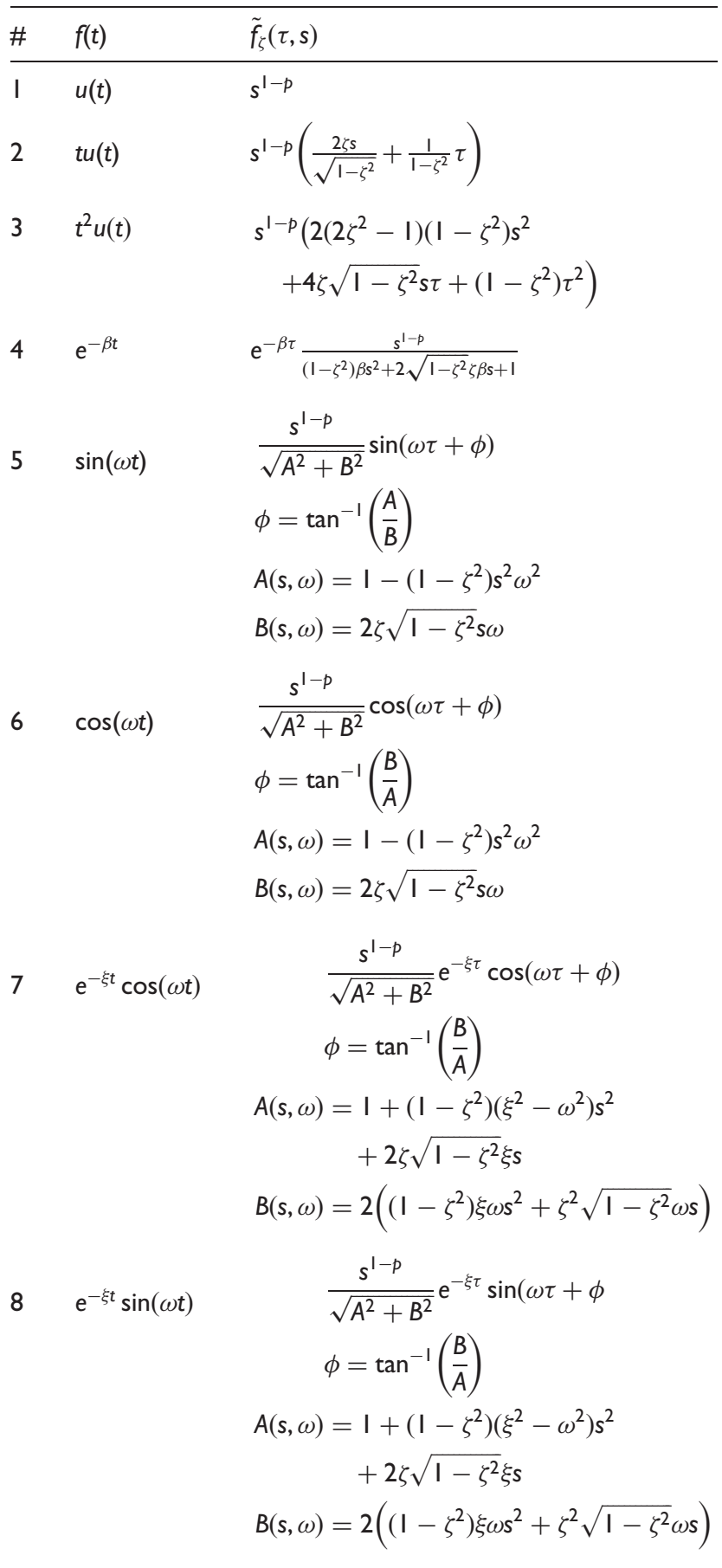

which can be evaluated by the integration by parts technique to obtain

$$
\begin{aligned}
\tilde{\dot{x}}_{\zeta}(\tau, s)= & \frac{s^{-p}}{1-\zeta^{2}}\left(\left.x(t) e^{\frac{-\zeta}{\sqrt{1-\zeta^{2}}}\left(\frac{t-\tau}{s}\right)} \sin \left(\frac{t-\tau}{s}\right)\right|_{\tau} ^{\infty}\right) \\
& -\frac{s^{-p}}{1-\zeta^{2}}\left(\int_{\tau}^{\infty} \frac{x(t)}{s \sqrt{1-\zeta^{2}}} e^{\frac{-\zeta}{\sqrt{1-\zeta^{2}}}\left(\frac{t-\tau}{s}\right)} \sin \left(\theta-\frac{t-\tau}{s}\right) \mathrm{d} t\right)
\end{aligned}
$$




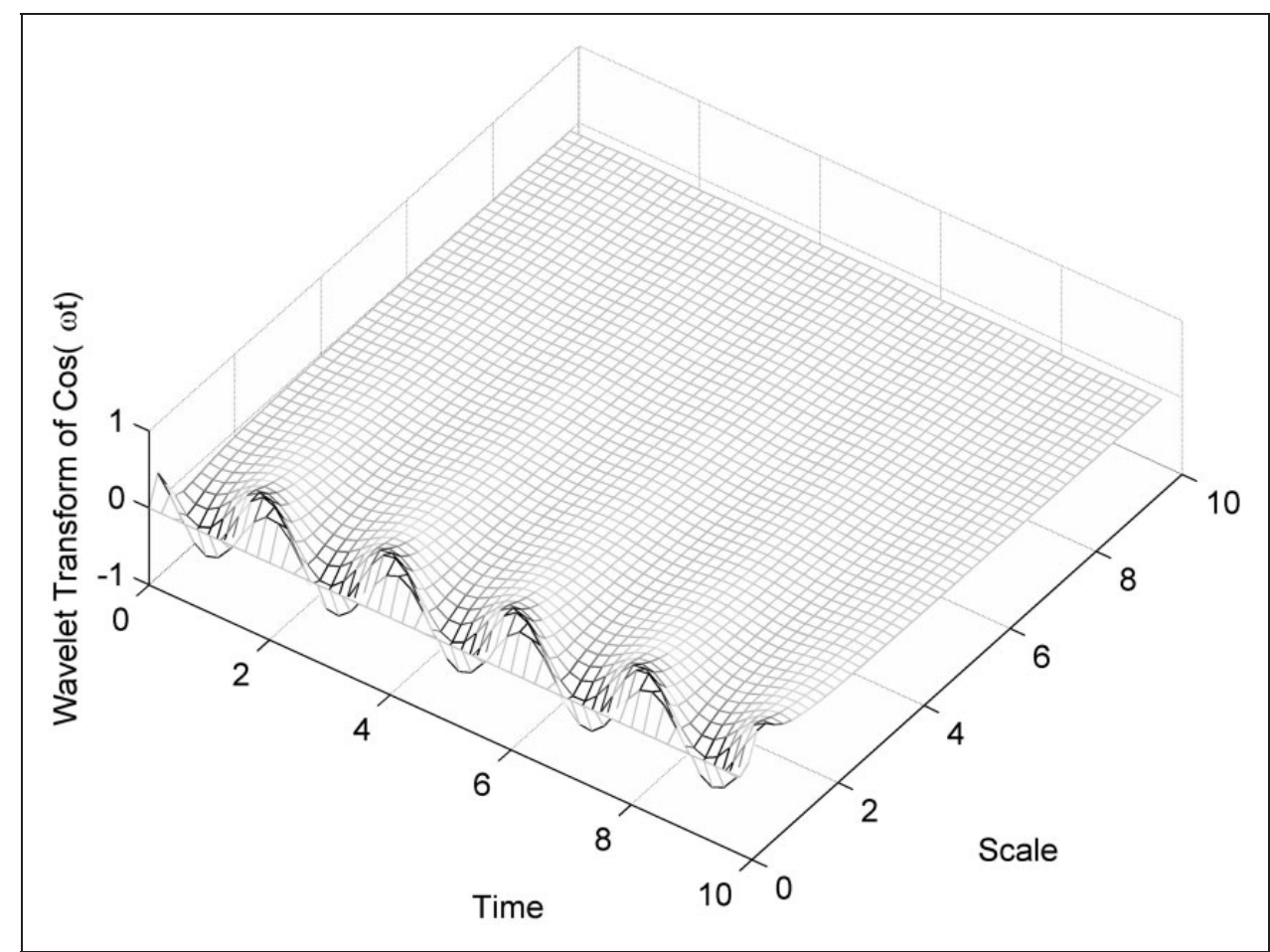

Figure 5. SOULTI wavelet transform of $f(t)=\cos (\omega t)$, at $\zeta=0.7$.

where $\theta$ is given by

$$
\theta=\tan ^{-1}\left(\frac{\sqrt{1-\zeta^{2}}}{\zeta}\right)
$$

Since $x(t)$ is exponentially bounded, the first term in equation (31) vanishes, so equation (31) becomes

$$
\begin{aligned}
\tilde{\dot{x}}_{\zeta}(\tau, s)= & \frac{e^{\frac{-\zeta}{\sqrt{1-\zeta^{2}}} \theta}}{s \sqrt{1-\zeta^{2}}} \\
& \times\left(\frac{s^{-p}}{1-\zeta^{2}} \int_{\tau}^{\infty} x(t) e^{\frac{-\zeta}{\sqrt{1-\zeta^{2}}}\left(\frac{t(\tau+s+s)}{s}\right)} \sin \left(\frac{t-(\tau+s \theta)}{s}\right) \mathrm{d} t\right)
\end{aligned}
$$

but the part inside the parenthesis is equal to $\tilde{x}_{\zeta}(\tau+s \theta, s)$, hence equation (33) is equivalent to equation (29).

Lemma 4. Let $J \subset(a, \infty) \subset \mathbb{R}$, and let $x(t): J \rightarrow \mathbb{R}$ be exponentially bounded as defined in Theorem 1, and the SOULTI wavelet transform of $x(t)$ be given by equation (10), then the SOULTI wavelet transform of $\chi(t)$, defined by

$$
\chi(t)=X(t)-X(a)=\int_{a}^{t} x(r) \mathrm{d} r
$$

is given by

$$
\begin{aligned}
\tilde{\chi}_{\zeta}(\tau, s) & =s \sqrt{1-\zeta^{2}} e^{\frac{\zeta}{\sqrt{1-\zeta^{2}}}} \tilde{x}_{\zeta}(\tau-s \theta, s)-s^{1-p} X(a) \\
\theta & =\tan ^{-1}\left(\frac{\sqrt{1-\zeta^{2}}}{\zeta}\right)
\end{aligned}
$$

Proof. Since $x(t)=\frac{\mathrm{d} X(t)}{\mathrm{d} t}$, substitute $x(t)$ in place of $\dot{x}(t)$, and $X(t)$ in place of $x(t)$ in equation (33), and the result can be written as

$\int_{\tau}^{\infty} x(t) \psi_{\zeta}\left(\frac{t-\tau}{s}\right) \mathrm{d} t=\frac{e^{\frac{-\zeta}{\sqrt{1-\zeta^{2}}} \theta}}{s \sqrt{1-\zeta^{2}}}\left(\int_{\tau}^{\infty} X(t) \psi_{\zeta}\left(\frac{t-(\tau+s \theta)}{s}\right) \mathrm{d} t\right)$

make the substitutions $\chi(t)+X(a)=X(t)$ and $\sigma=\tau+s \theta$ into equation (36), then equation (35) follows.

\section{The time-frequency resolution and properties}

The time-frequency resolution is an important property of the wavelet transform. The time-frequency 
resolution is defined by

$$
\mu=\Delta T \Delta \Omega
$$

where $\Delta T$ is the time resolution or the time window and $\Delta \Omega$ is the frequency resolution or frequency window. The time window represents the time interval that a frequency can be identified within, while $\Delta \Omega$ represents the range of frequencies within a time interval. There are different ways to define the time and frequency resolutions. One possible way is to use the standard deviation definition, in which the resolutions are defined by

$$
\begin{gathered}
\left(\frac{\Delta T_{S D}}{2}\right)^{2}=\frac{\int_{-\infty}^{\infty}\left(t-t_{C G \zeta}\right)^{2}\left|\psi_{\zeta}(t)\right|^{2} \mathrm{~d} t}{\int_{-\infty}^{\infty}\left|\psi_{\zeta}(t)\right|^{2} \mathrm{~d} t} \\
\left(\frac{\Delta \Omega_{S D}}{2}\right)^{2}=\frac{\int_{-\infty}^{\infty}\left(\omega-\omega_{C G \zeta}\right)^{2}\left|\Psi_{\zeta}(\omega)\right|^{2} \mathrm{~d} \omega}{\int_{-\infty}^{\infty}\left|\Psi_{\zeta}(\omega)\right|^{2} \mathrm{~d} \omega}
\end{gathered}
$$

where $t_{C G \zeta}$ and $\omega_{C G \zeta}$ represent the center of mass of the signal in time and frequency respectively, and they are given by

$$
\begin{gathered}
t_{C G \zeta}=\frac{\int_{0}^{\infty} t\left|\psi_{\zeta}(t)\right|^{2} \mathrm{~d} t}{\int_{0}^{\infty}\left|\psi_{\zeta}(t)\right|^{2} \mathrm{~d} t} \\
\omega_{C G \zeta}=\frac{\int_{0}^{\infty} \omega\left|\Psi_{\zeta}(\omega)\right|^{2} \mathrm{~d} \omega}{\int_{0}^{\infty}\left|\Psi_{\zeta}(\omega)\right|^{2} \mathrm{~d} \omega}
\end{gathered}
$$

Table 2 lists the results of calculating $\mu$ for some values of $0<\zeta<1$ at $p=1$. The values of $\mu$ do not depend on the scale value and they satisfy the Heisenberg principle (Kaiser, 1994). Using the standard deviation, the resolution satisfies the inequality $\mu>1 / 4 \pi$ (Kaiser, 1994). $\omega_{C G \zeta}$ is proportional to the scale $s$, while $t_{C G \zeta}$ is inversely proportional to $s$. However, the standard deviation does not offer meaningful time and frequency windows of resolution. The SOULTI wavelet is not symmetrical neither in time nor in frequency. Moreover, it has no compact support neither in time nor in frequency. So we would question the significance of the standard deviation window about the signal center in time and the significance of the frequencies included in the standard deviation window and weather that is really what is accentuated in the time-scale or time-frequency analysis.

We can attain an alternative definition for the SOULTI wavelet time-frequency resolution based on systems dynamics and control theory. The system response is considered settled when it enters the $2 \%$ margin of the final value and never leaves it again. Therefore, we can use the $2 \%$ settling-time value to define the time window, namely $\Delta T_{2 \%}=2 \% t_{s t}$.
Table 2. Time-frequency resolution based on the standard deviation definition at $p=\mathrm{I}$.

\begin{tabular}{llllll}
\hline$\zeta$ & $\mathrm{t}_{\mathrm{CG} \zeta}$ & $\omega_{\mathrm{CG} \zeta}$ & $\Delta \mathrm{T}_{\mathrm{SD}}$ & $\Delta \Omega_{\mathrm{SD}}$ & $\mu$ \\
\hline 0.05 & 20.075 & $0.4853 \mathrm{I}$ & 39.85 & 0.24577 & 9.794 \\
0.1 & 10.149 & 0.47298 & 19.704 & 0.33949 & 6.689 \\
0.15 & 6.8878 & 0.46292 & 12.896 & 0.40724 & 5.252 \\
0.2 & 5.2909 & 0.45506 & 9.4305 & $0.4619 \mathrm{I}$ & 4.356 \\
0.25 & 4.3571 & 0.44939 & 7.3101 & 0.5088 & 3.719 \\
0.3 & 3.7522 & 0.44595 & 5.8715 & 0.55084 & 3.234 \\
0.35 & 3.3322 & 0.44484 & 4.8325 & 0.58997 & 2.851 \\
0.4 & 3.0245 & 0.44625 & 4.0534 & 0.62763 & 2.544 \\
0.45 & 2.7882 & 0.45046 & 3.4579 & 0.66502 & 3 \\
0.5 & $2.598 \mathrm{I}$ & 0.4579 & 3 & 0.7033 & 2.11 \\
0.55 & 2.4372 & 0.46922 & 2.6493 & 0.74366 & 1.970 \\
0.6 & 2.2933 & 0.48537 & 2.3828 & 0.78752 & 1.877 \\
0.65 & 2.157 & 0.50783 & 2.1798 & 0.83668 & 1.824 \\
0.7 & 2.02 & 0.53899 & 2.0203 & 0.89372 & 1.806 \\
0.75 & 1.8741 & 0.58291 & 1.8838 & 0.96259 & 1.813 \\
0.8 & 1.71 & 0.64715 & 1.7483 & 1.05 & 1.836 \\
0.85 & 1.5153 & 0.74778 & 1.5888 & 1.1692 & 1.858 \\
0.9 & 1.2689 & 0.927 & 1.3714 & 1.3512 & 1.853 \\
0.95 & 0.92196 & 1.3553 & 1.0296 & 1.7057 & 1.756 \\
\hline & & & & &
\end{tabular}

In the frequency domain, the frequency corresponding to attenuating the input power by a half is considered the frequency bandwidth of the system or the cut-off frequency, so we can use the bandwidth to define the frequency window.

The $2 \%$ settling time, $t_{s t}$ is reached when the enveloping function enters within $2 \%$ of the final value. Therefor, for a scaled wavelet, it is given by

$$
t_{s t}=-\frac{s \sqrt{1-\zeta^{2}}}{\zeta} \log (0.02) \simeq \frac{4 s \sqrt{1-\zeta^{2}}}{\zeta}
$$

On the other hand, the wavelet bandwidth is the frequency at which the frequency spectrum of a scaled wavelet satisfies

$$
|s \Psi(s \omega)|^{2}=\frac{\frac{s^{2}-2 p}{1-\zeta^{2}}}{\left(-s^{2} \omega^{2}+\frac{1}{1-\zeta^{2}}\right)^{2}+\left(\frac{2 \zeta \omega s}{\sqrt{1-\zeta^{2}}}\right)^{2}}=\frac{1}{2}
$$

Solving for $\omega$ gives the bandwidth by

$$
\begin{aligned}
\left.\omega\right|_{|s \Psi(s \omega)|^{2}=\frac{1}{2}} & =\Delta \Omega_{B W} \\
& =\frac{1}{s \sqrt{1-\zeta^{2}}} \sqrt{1-2 \zeta^{2}+\sqrt{2 s^{2-2 p}-4 \zeta^{2}+4 \zeta^{4}}}
\end{aligned}
$$


Substitute equation (42) and equation (44) into equation (37) gives the resolution as

$$
\mu=4 \frac{\sqrt{1-2 \zeta^{2}+\sqrt{2 s^{2-2 p}-4 \zeta^{2}+4 \zeta^{4}}}}{\zeta}
$$

Equation (45) gives us a way to determine an appropriate value for $p$ based on the time-frequency resolution shape. In order for $\mu$ to be independent of $s$, we must have $p=1$, which yields

$$
\mu(\zeta)=4 \frac{\sqrt{1-2 \zeta^{2}+\sqrt{2-4 \zeta^{2}+4 \zeta^{4}}}}{\zeta}
$$

which indicates that the $2 \% t_{s t}-B W$ resolution definition depends only on $\zeta$ thus on the wavelet family $\psi_{\zeta}$, so we may write the resolution as $\mu\left(\psi_{\zeta}\right)$.

Now, let us investigate the values of $\mu$ in range $0<\zeta<1$. When $\zeta \rightarrow 0$, we have

$$
\lim _{\zeta \rightarrow 0} \mu(\zeta)=\infty
$$

while when $\zeta \rightarrow 1$ we get

$$
\lim _{\zeta \rightarrow 1} \mu(\zeta)=2.65
$$

Since $\frac{d \mu}{d \zeta}<0 \quad \forall \quad \zeta \in(0,1)$, then

$$
\frac{\pi}{4}<2.65<\mu(\zeta)<\infty
$$

which means that the $2 \% t_{s t}-B W$ definition satisfies the Heisenberg principle when $p=1$. Another advantage of having $p=1$, is preserving the wavelet frequency function peak constant. This is sometimes useful since it guarantees that all the frequency bands are amplified at the same level, see Figure 6. This functions as a normalizing factor though it does not preserve the wavelet energy. Figure 2 shows the standard deviation-based and the $2 \% t_{s t}$ time windows, while Figure 3 illustrates the standard deviation-based and the half-power bandwidth-based frequency windows for the SOULTI wavelet.

The $2 \% t_{s t}-B W$ gives a better meaning for the timefrequency resolution of the SOULTI wavelet, but when $\zeta$ is small, $\zeta<0.4$, the definition suffers from two problems. First, the variation in the frequency response magnitude varies significantly within the bandwidth, which requires better focus on the resonance range. Secondly, as $s$ decreases, the bandwidth of $\psi\left(\frac{t}{s}\right)$ contains all the bandwidths corresponding to larger $s$, i.e. $\Delta \Omega_{B W}\left(\zeta, s_{2}\right) \subset \Delta \Omega_{B W}\left(\zeta, s_{1}\right)$ when $s_{1}<s_{2}$.

For $\zeta<0.4$, another definition of the time-frequency resolution, that better reflects the data on the time-scale or the time-frequency analysis domain can be provided

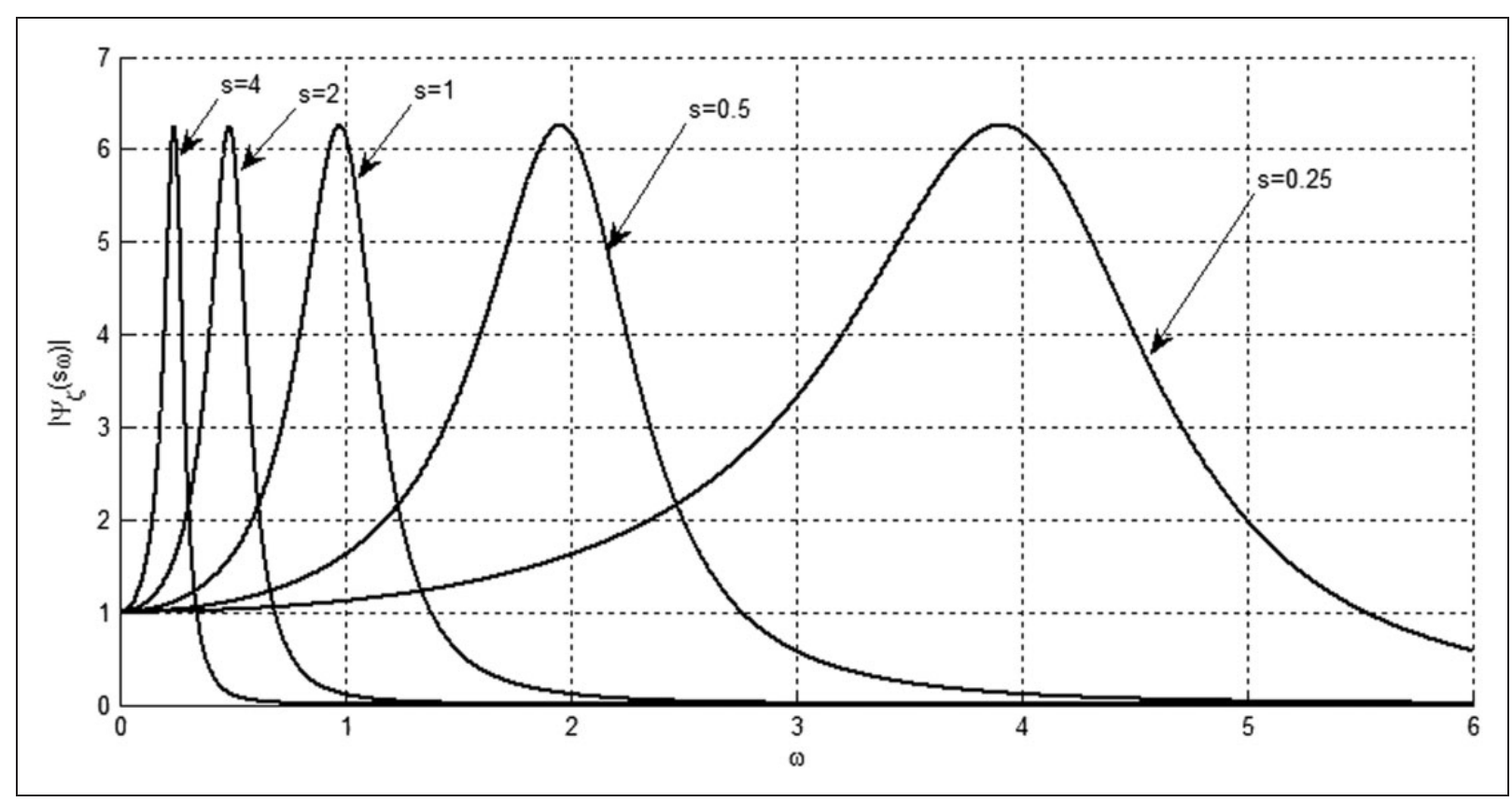

Figure 6. Wavelet amplitude in frequency domain for different values of the scale $s$ at $\zeta=0.2$. 
based on the quality factor half-power bandwidth definition. The quality factor is the peak value of the frequency response. For small $\zeta$, the quality factor for LTI second-order system can be approximated by (Meirovitch, 1997)

$$
Q=\frac{1}{2 \zeta}
$$

The half power points, $q_{1}$ and $q_{2}$ are the points when $|\Psi(s \omega)|=\frac{Q}{\sqrt{2}}$, see Figure 7. The bandwidth of the frequency response is

$$
\Delta \Omega_{Q}=\omega_{2}-\omega_{1}
$$

where $\omega_{1}$ is the corresponding frequency to $q_{1}$, and $\omega_{2}$ is the corresponding frequency to $q_{2}$, as shown in Figure 7. To find $\omega_{1}$ and $\omega_{2}$, we have to solve the wavelet power in equation (52) for $\omega$ where $p=1$

$$
|\Psi(s \omega)|^{2}=\frac{1}{\left(-s^{2} \omega^{2}\left(1-\zeta^{2}\right)\right)^{2}+\left(2 \zeta \omega \sqrt{1-\zeta^{2}}\right)^{2}}=\frac{1}{8 \zeta^{2}}
$$

From equation (52) we find

$$
\begin{aligned}
& \omega_{1}^{2}=\frac{1-2 \zeta}{s^{2}\left(1-\zeta^{2}\right)} \\
& \omega_{2}^{2}=\frac{1+2 \zeta}{s^{2}\left(1-\zeta^{2}\right)}
\end{aligned}
$$

Using the approximation $\left(\omega_{1}+\omega_{2} \simeq \frac{2}{s \sqrt{1-\zeta^{2}}}\right)$, which is valid for small values of $\zeta$, it is easy to show that

$$
\Delta \Omega_{Q}=\omega_{2}-\omega_{1}=\frac{2 \zeta}{s \sqrt{1-\zeta^{2}}}
$$

Substituting equations (42) and (55) into equation (37), the new time-frequency resolution definition becomes

$$
\mu=\Delta T \Delta \Omega \simeq \frac{4 s \sqrt{1-\zeta^{2}}}{\zeta} \frac{2 \zeta}{s \sqrt{1-\zeta^{2}}}=8
$$

Equation (56) shows a very interesting result where the time-frequency resolution is constant and does not

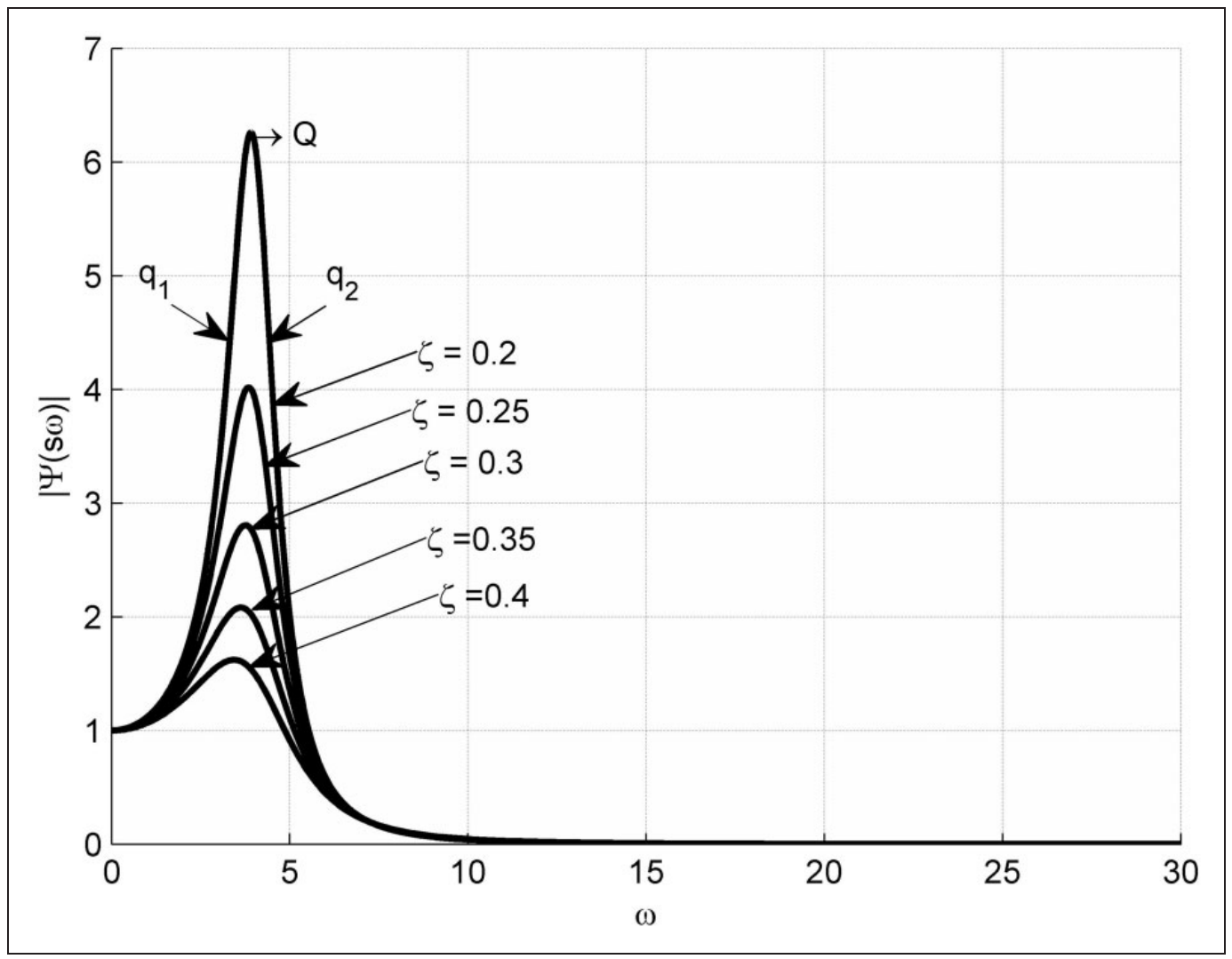

Figure 7. $|\Psi(s \omega)|$ for different values of $\zeta$ showing the quality factor and the half-power bandwidth. 
depend on $\zeta$. Note that this approximation is valid for values of $\zeta<0.4$, for larger values the quality factor is smaller, hence the time-frequency resolution defined by equations (45) and (46) would be more meaningful and suitable to adopt. Of course one may require a wider bandwidth than the half-power quality factor bandwidth, which will make the time-frequency resolution coarser. For example, if instead of the $\frac{Q}{\sqrt{2}}$ bandwidth limit we use $Q / x$, where $x<Q$, then the bandwidth and the time-frequency resolution become

$$
\begin{aligned}
\Delta \Omega_{Q} & =\simeq \frac{2 \sqrt{x^{2}-1} \zeta}{s \sqrt{1-\zeta^{2}}} \\
\mu & =8 \sqrt{x^{2}-1}
\end{aligned}
$$

The frequency at $Q$ represents the frequency at which the wavelet filter is centered at. Moreover, it is easy to predict where the wavelet frequency is centered because the scale is directly linked to frequency as stated by equation (6). The peak occurs at (Meirovitch, 1997)

$$
\omega_{Q}=\omega_{n} \sqrt{1-2 \zeta^{2}}
$$

For small $\zeta$ we have

$$
\omega_{Q}=\omega_{n} \sqrt{1-2 \zeta^{2}} \simeq \omega_{n} \sqrt{1-\zeta^{2}}=\omega_{d}
$$

which with equation (6) shows that we can easily approximate to a good accuracy the wavelet peak frequency by the relation

$$
\omega_{Q} \simeq \frac{1}{S}
$$

Figure 6 shows clearly the accuracy of equation (61) for the $\psi_{0.2}\left(\frac{t}{s}\right)$ SOULTI family with different scaling values. For larger values of $\zeta$, i.e. $\zeta>=0.4$, the approximation in equation (61) is not valid and we have to use the exact relation

$$
\omega_{Q}=\frac{\sqrt{1-2 \zeta^{2}}}{\sqrt{1-\zeta^{2}}} \frac{1}{S}
$$

The SOULTI wavelet has $\Psi(0)=1$ when $p=1$. For other values of $p$, the wavelet magnitude depends on $s^{1-p}$. Moreover, we have

$$
\int_{0}^{\infty} \frac{\left|\Psi_{\zeta}(\omega)\right|^{2}}{\omega} \mathrm{d} \omega=\infty
$$

which implies that the SOULTI wavelet does not satisfy the admissibility condition stated in equation (64) but it has an inverse

$$
0<\int_{0}^{\infty} \frac{|\Psi(\omega)|^{2}}{\omega} \mathrm{d} \omega=C<\infty
$$

\section{Application: Frequency identification and spectrogram}

To validate the capability of the SOULTI wavelet in detecting features of signals, we produce the frequency evolution or the scale evolution with respect to time of some signals using the SOULTI wavelet. We present some examples of SOULTI wavelet analysis of finite time signals with white noise added to them at different Signal to Noise Ratio $(S N R)$ levels.

It is important to emphasize that the continuous version of the wavelet transform is performed in these examples, where the transform integral is performed numerically. In all the examples, $\zeta=0.1$ is used because it gives the wavelet a large quality factor value as shown in Figure 6.

\section{I. Identifying constant frequencies in time-invariant frequency signals}

First, two noisy signals with the same frequency are tested. The first has $S N R=15 \mathrm{~dB}$, and the second has $S N R=$ $7.5 \mathrm{~dB}$. Figure 8 shows the two signals. Figure 9 shows the contour map of the two signals wavelet transform.

We notice that in both cases the ridges and the peaks are distinctly recognized at $s=\frac{1}{2}$, which corresponds to $\omega=2 \mathrm{rad} / \mathrm{s}$ by the scale-frequency relation in equation (6). The ridge of the wavelet transform is the set of points in the time-scale domain $\Theta$, where the wavelet integral has stationary points $(t, s) \in \Theta$ such that $t_{s}(t, s)=s$, where $t_{s}$ is a stationary point, i.e. $\left.\frac{\mathrm{d} \tilde{f}_{\xi}(\tau, s)}{d s}\right|_{t_{s}}=0 \quad$ (Tchamitchan and Torresani, 1992). Notice also that at the end of the signal the transform is distorted and the peaks diminish due to the edge effect. Also notice that since the SOULTI wavelet is causal the edge effect appears at the end of the time scale of the signal only and the noisy signal with $S N R=7.5 \mathrm{~dB}$ has slightly worse edge effect.

In the second test, a signal carrying two different frequencies is analyzed. The signal has $S N R=15 \mathrm{~dB}$ and is graphed in Figure 10. Figure 11 shows two mappings. The first maps the contours on the time-scale domain and it shows clearly two ridges that stretch along two lines of constant scale $s=0.125$ and $s=0.5$, parallel to the time axis. The second plots the contours on the time-frequency domain. The scale-frequency conversion is performed using equation (6). The ridges stretch along the constant frequency values $\omega=2 \mathrm{rad} / \mathrm{s}$ and $\omega=8 \mathrm{rad} / \mathrm{s}$. 


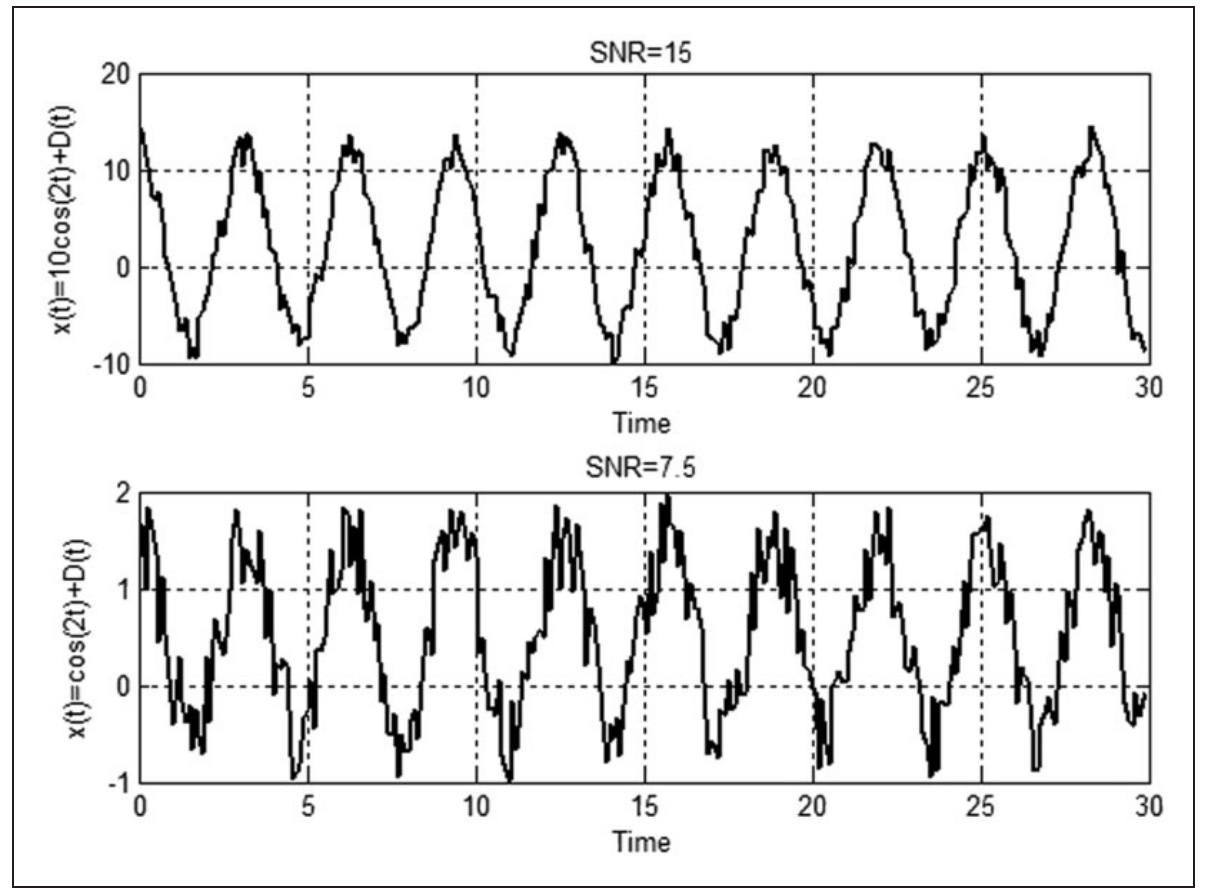

Figure 8. Top: single harmonic with white noise of $S N R=15 \mathrm{~dB}$. Bottom: single harmonic with white noise of $S N R=7.5 \mathrm{~dB}$.

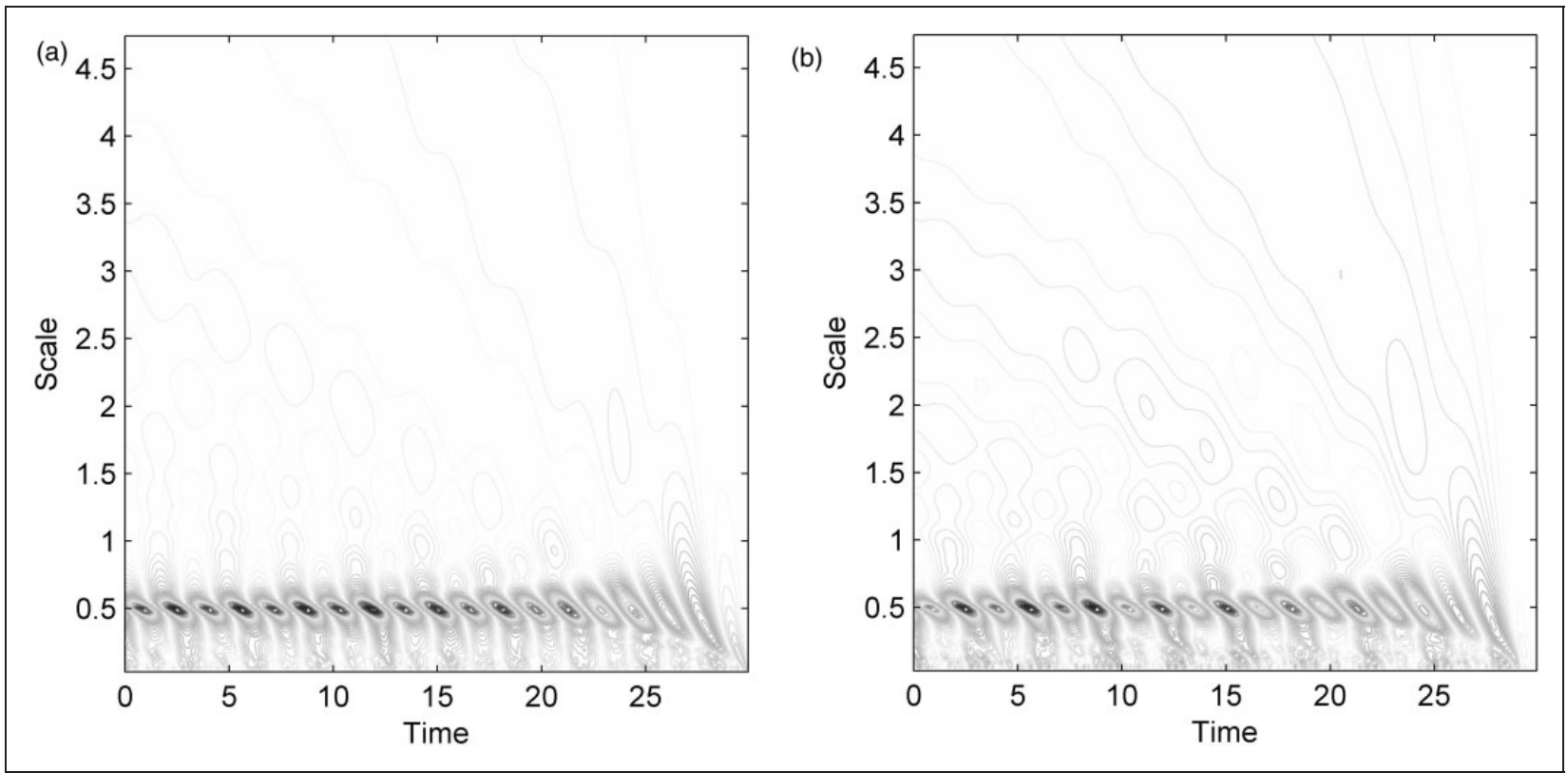

Figure 9. Contour mapping of the SOULTI wavelet Transform of a harmonic signal of frequency $=2 \mathrm{rad} / \mathrm{s} ;$ (a) $S N R=15 \mathrm{~dB}$, (b) $S N R=7.5 \mathrm{~dB}$.

\subsection{Identifying the instantaneous frequency in time-varying frequency signal}

The advantage of the time-scale or time-frequency analysis over classic frequency analysis is that it is more useful in analyzing time-varying and nonlinear oscillations. In this example, we analyze a signal consisting of a combination of constant harmonics with linear chirp as a time-varying frequency component. White noise is added to the signal with $S N R=20 \mathrm{~dB}$. The signal is 


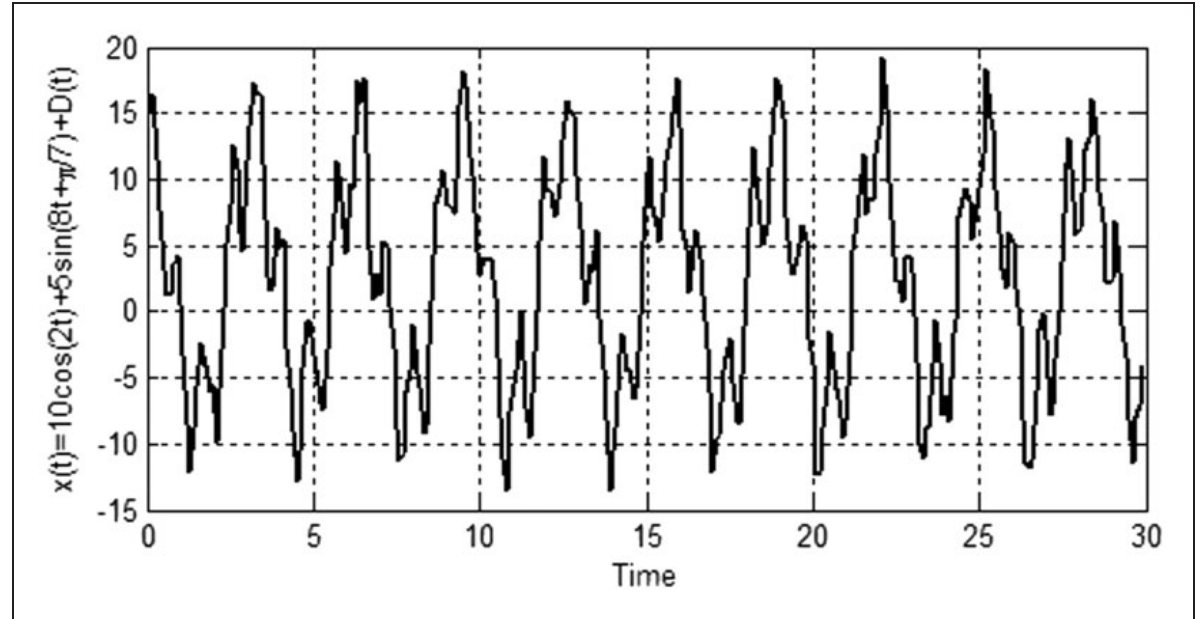

Figure 10. Sum of two harmonics with white noise. $\omega_{1}=2 \mathrm{rad} / \mathrm{s}, \omega_{2}=8 \mathrm{rad} / \mathrm{s}$, and the $S N R=15 \mathrm{~dB}$.

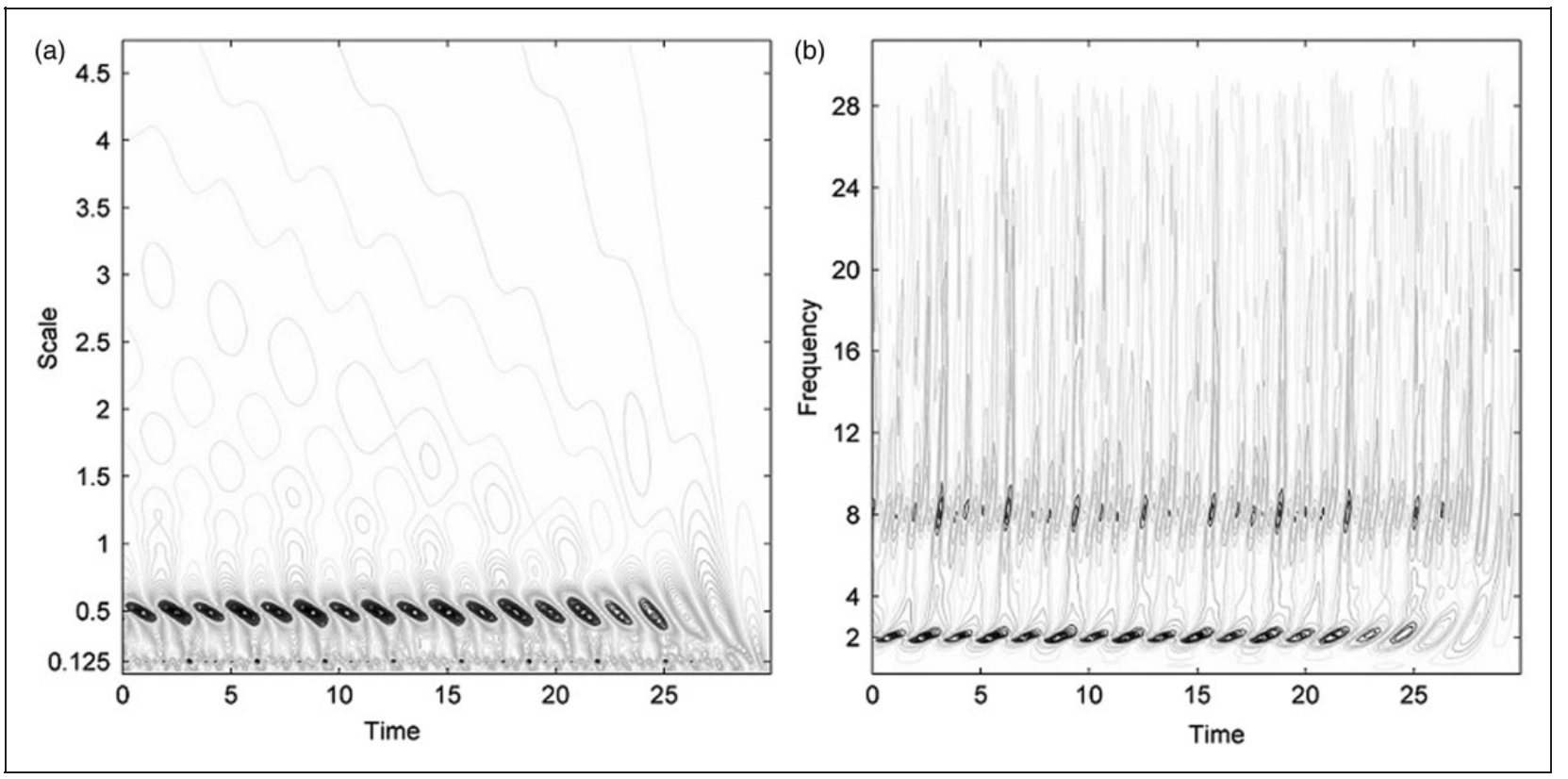

Figure II. SOULTI wavelet transform for the two harmonics signal in Figure I0. (a) Time-scale contour mapping. (b) Timefrequency contour mapping.

given by equation (65)

$$
x(t)=10 \sin \left(0.4 t^{2}\right)+5 \cos (2 t)+8 \sin \left(t+\frac{\pi}{7}\right)+D(t)
$$

where $D(t)$ represents the white noise or the disturbance term. Figure 12 plots the signal in the time domain. Performing Fourier analysis to the signal does not reveal the instantaneous frequency change in the signal. Figure 13 shows the Fast Fourier Transform
(FFT) and the Welch averaging of the frequency spectrum. While the FFT identifies the constant harmonics with peaks at $\omega=1$ and $\omega=2$, it is not possible to distinguish the instantaneous frequency change from the FFT. The Welch averaging does not identify the constant harmonics because of the interference from the frequency-changing component.

Figure 14 shows the SOULTI wavelet transform of the signal. The transform distinctly traces the instantaneous frequency with respect to time, where the dashed lines represents ridgelines that trace this 


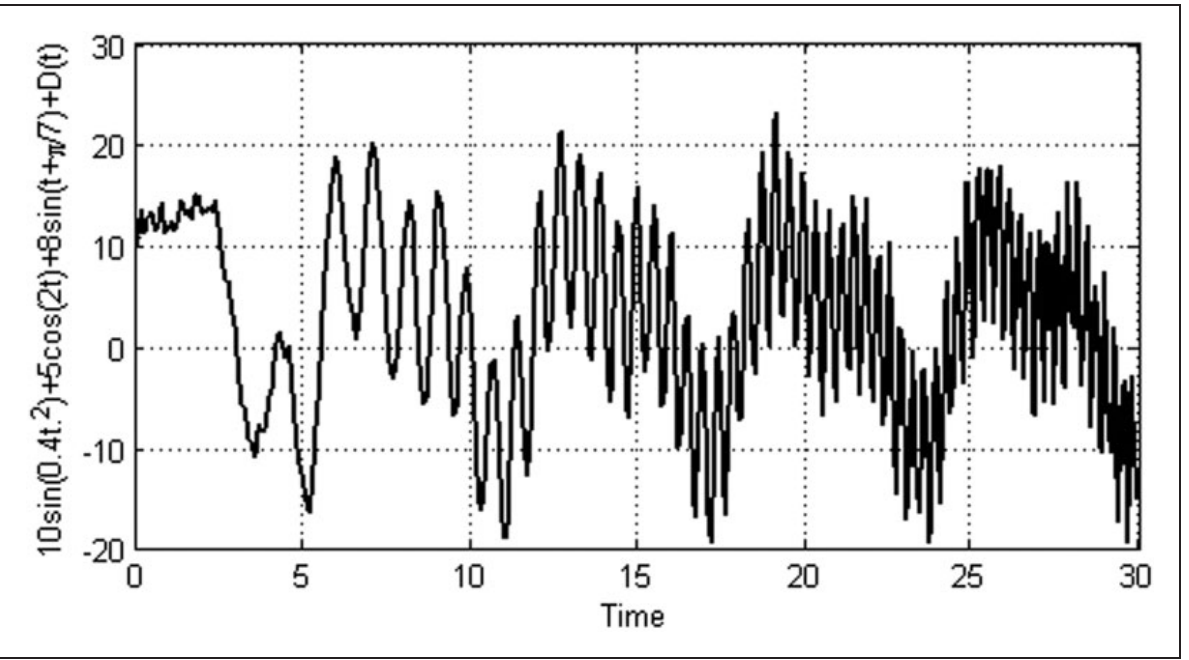

Figure 12. Two constant harmonics with a time varying frequency component signal.

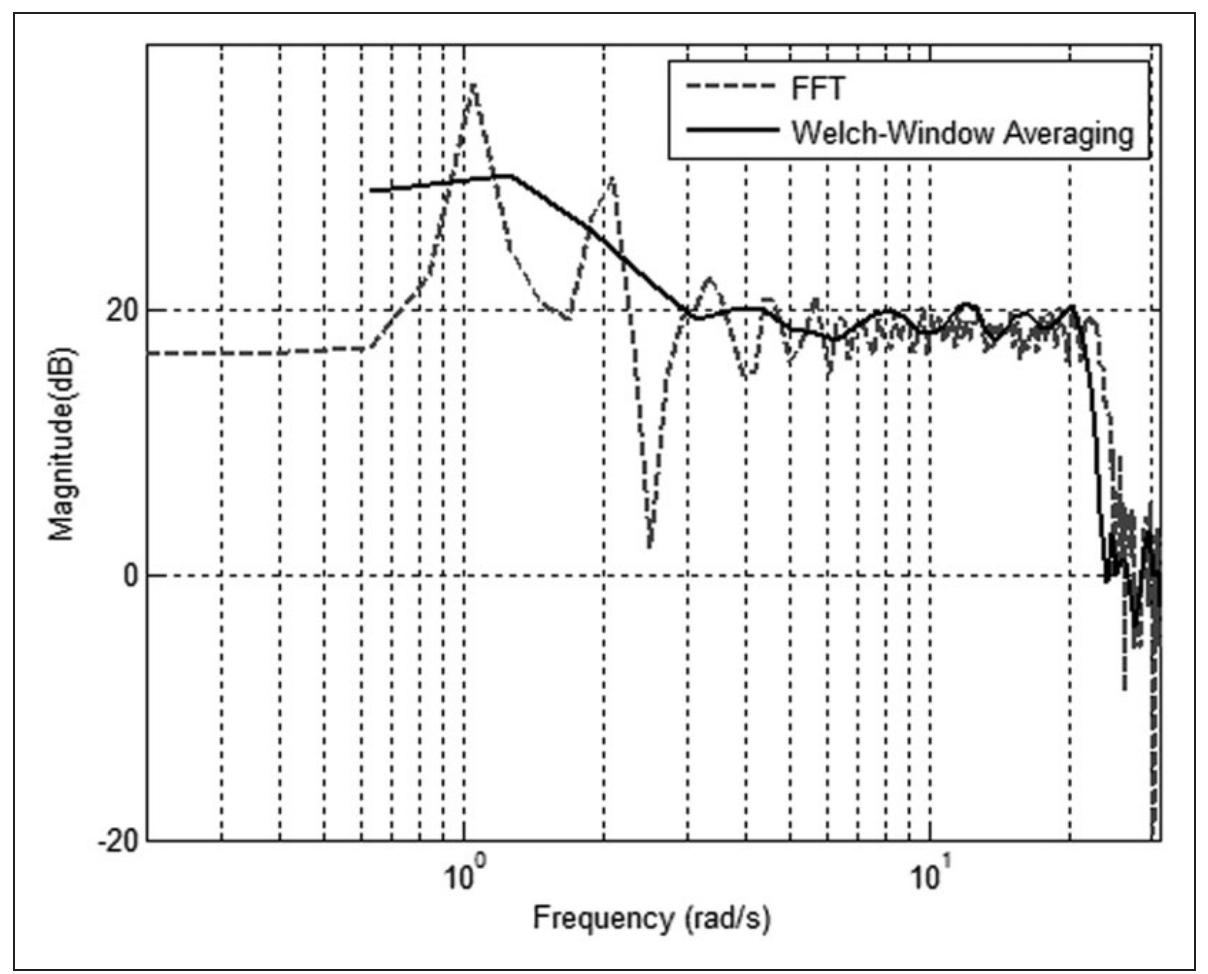

Figure 13. Frequency spectrum of the signal described in Figure 12. (Dashed line) FFT. (Solid line) Welsh spectrum averaging.

frequency along time. When examining the time varying component in equation (65) we find that the instantaneous frequency is given by $\omega(t)=0.8 t$, which is the equation of the dashed line on the time-frequency wavelet mapping shown in Figure 14(b). The dashed curve in Figure $14(\mathrm{a})$ is the inverse of the line $\omega=0.8 t$, namely $s(t)=1 / 0.8 t$, which conforms to the scale-frequency relation in equation (6). Notice also, that the other two constant frequencies are identified along ridgelines of almost constant scales at $s=0.5$ and $s=1$ in Figure 14(a) and along ridgelines of almost constant frequency at $\omega=1$ and $\omega=2$ in Figure 14(b). On 14(a), the parabolic dashed line, which traces the instantaneous change of the chirp frequency, intersects the $s=1$ and the $s=0.5$ lines at times $t=0.26 \mathrm{~s}$ and $t=1.6 \mathrm{~s}$ respectively.

As a comparison between the SOULTI wavelet and other wavelets in resolving frequencies with respect to 


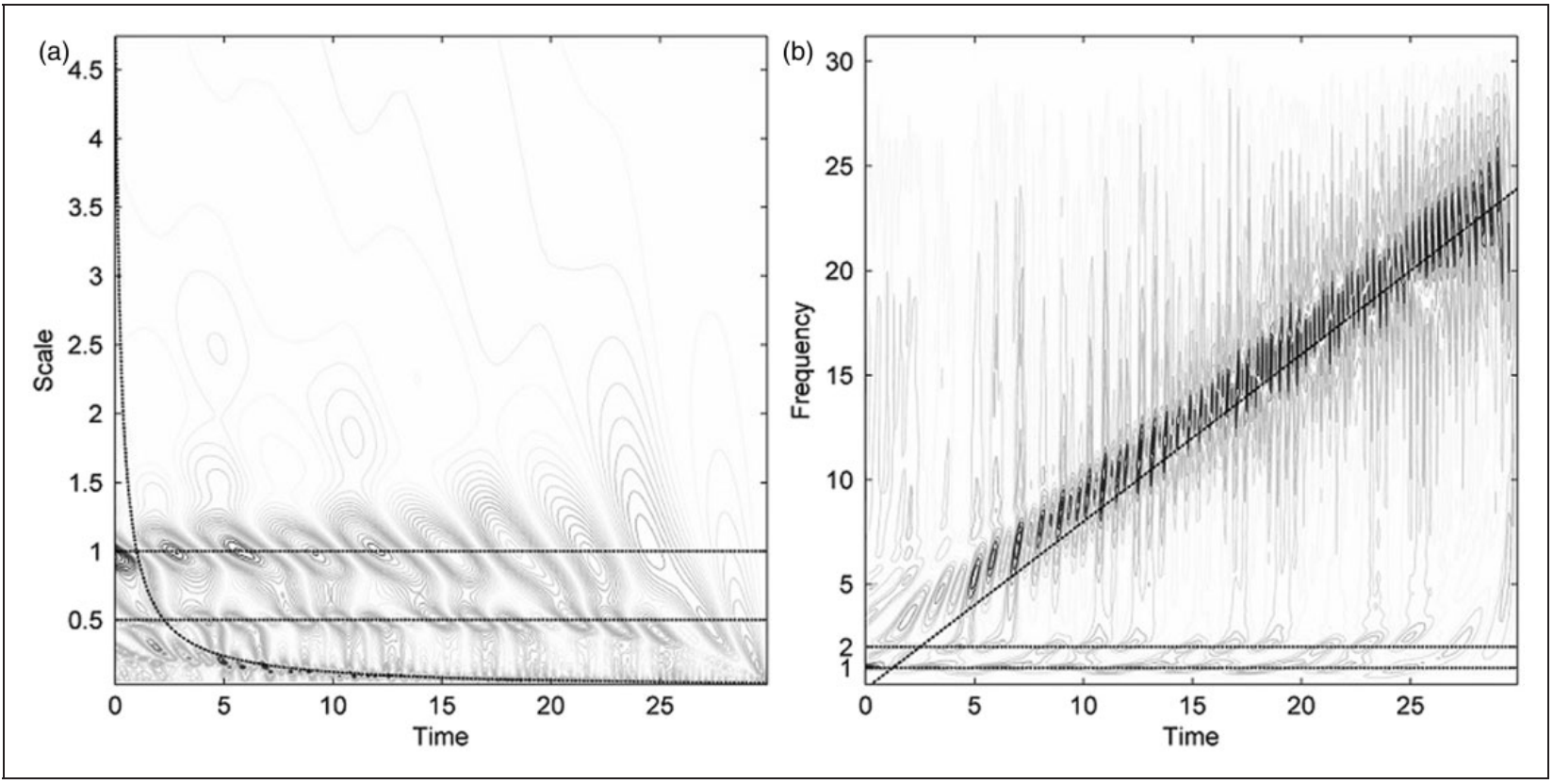

Figure 14. SOULTI wavelet transform for the chirp signal described in Figure 12 and shown in Figure I2. (a) Scalogram (Time-scale) contour mapping (b) Spectrogram (Time-frequency) contour mapping.

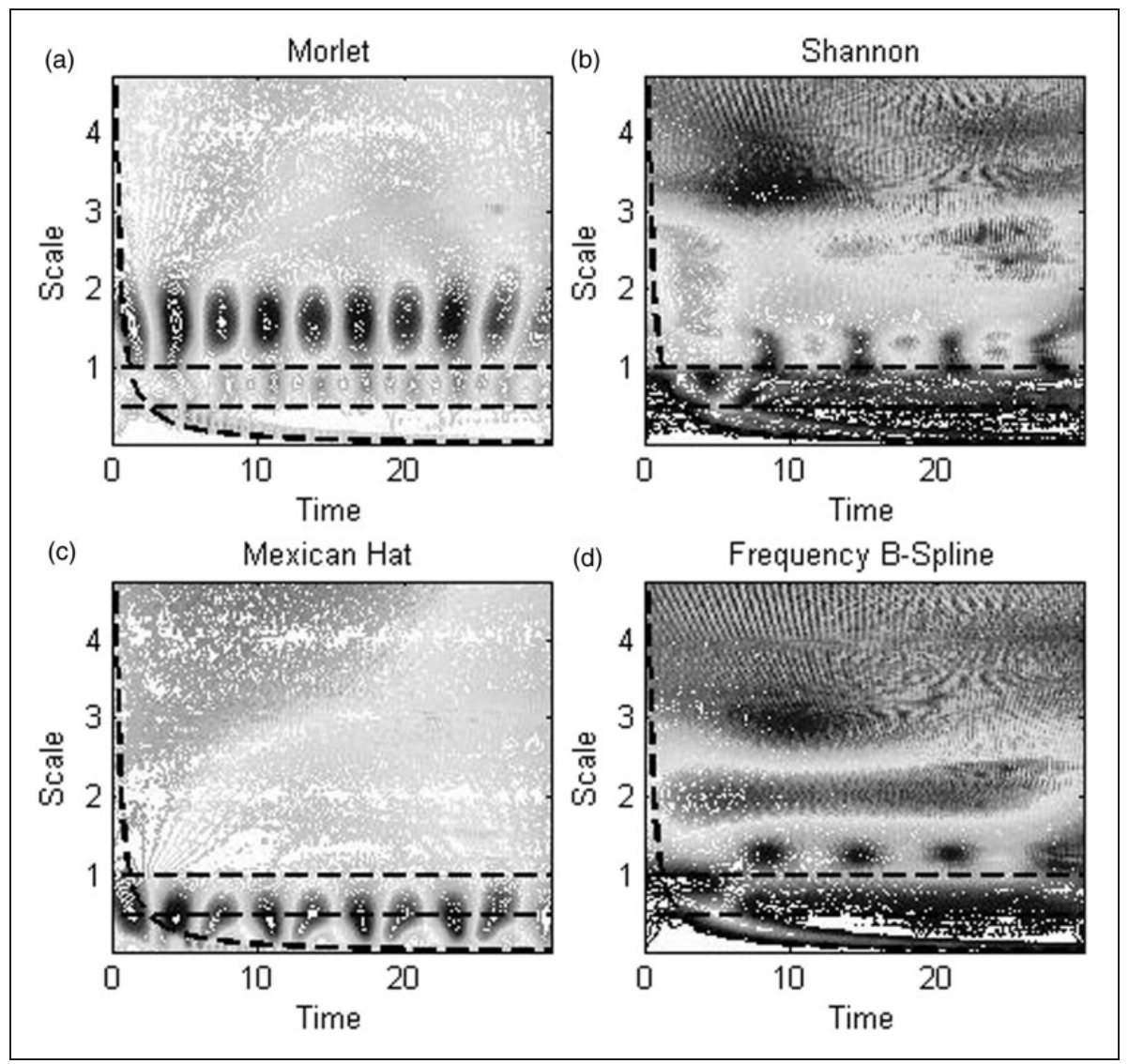

Figure 15. Scalograms of the signal described in Figure 12 using different wavelets. (a) By Morlet wavelet; (b) by Complex Shannon wavelet $\left(f_{b}=I, f_{c}=I\right)$; (c) by Mexican hat wavelet; (d) by Frequency B-Spline wavelet (order $\left.=2, f_{b}=I, f_{c}=I\right)$. $f_{b}$ : bandwidth frequency. $f_{c}$ : wavelet center frequency. 


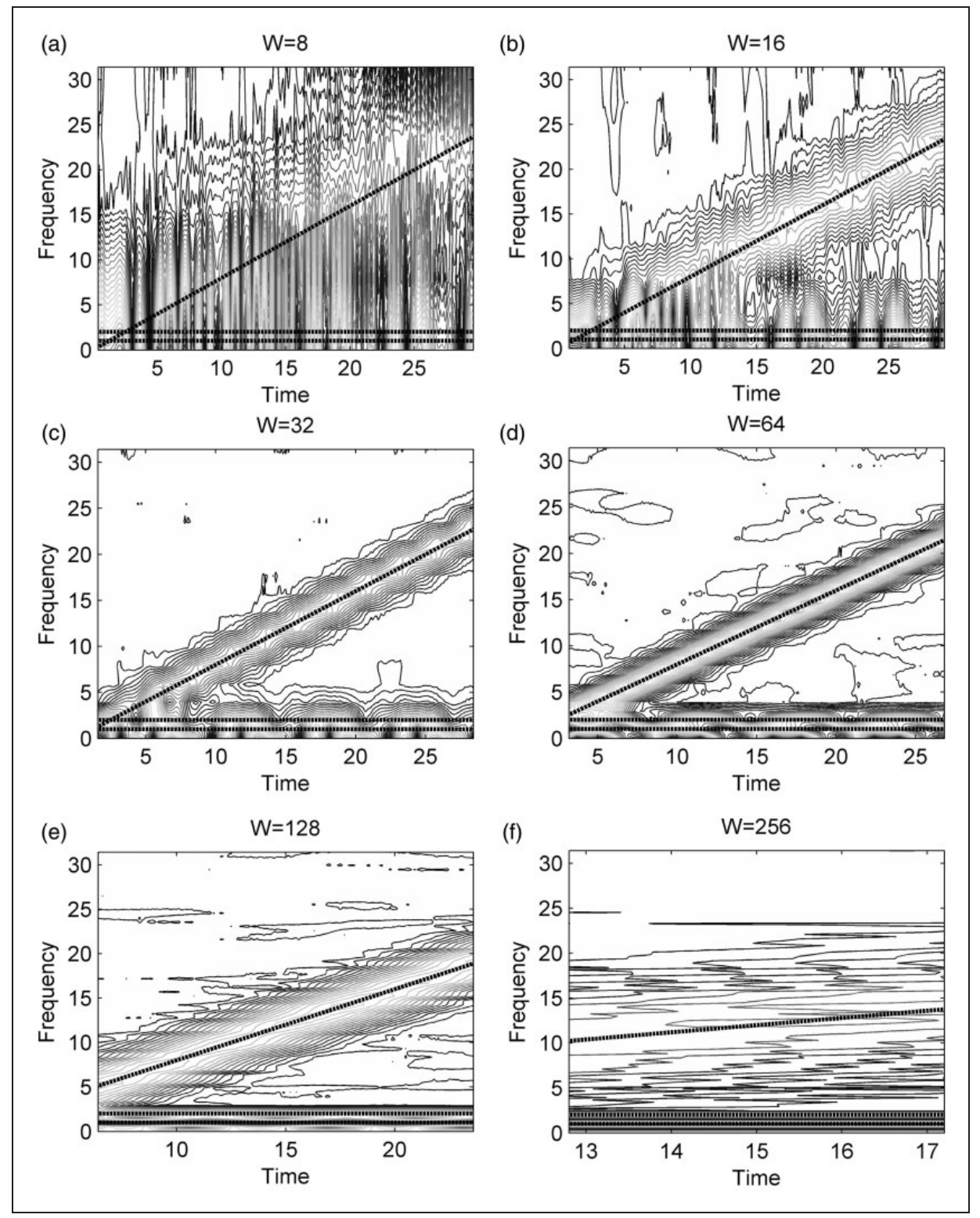

Figure 16. Spectrograms of the linear chirp signal in Figure 12 at different Window widths (samples). (a) $W=8$ (b) $W=16$ (c) $W=32$ (d) $W=64$ (e) $W=128$ (f) $W=256$.

time, the same chirp signal is analyzed with four different wavelets, Morlet, complex Shannon, Mexican hat and the frequency B-Spline. The four wavelets scalogram graphs are shown in Figure 15. Notice that the four are able to resolve the chirp into curves parabolic in shape with different ridge widths. However, the curves are not the reciprocal of the instantaneous frequency. In addition, the constant scale ridges are not at scales that can be easily matched to frequencies.

The Mexican hat wavelet gives the best match to the parabolic curve, but when resolving the constant frequencies in the signal it shows large shifts. It is difficult to infer the accurate frequencies in the signal from these wavelets scalogram maps, though one can infer qualitative information about the shape of the instantaneous frequency change with respect to time. For each wavelet, the relation between the scale and the frequency along the ridgeline is different, but one can argue that it is the reciprocal of some function of the instantaneous frequency.

From the previous discussion, we conclude that it is difficult to construct a spectrogram for each scalogram shown in Figure 15. However the SOULTI wavelet scalogram can be directly transformed into spectrogram by applying the scale-frequency change in equation (6).

To evaluate the SOULTI wavelet spectrogram, we compare it to the Short Time Fourier Transform 
(STFT) spectrograms. Six spectrograms based on the STFT were computed for different window sizes $(W)$, where the size is measured by the number of samples. To make the comparison compatible with the continuous wavelet transform, the window overlap is set as $(W-1)$ to perform window sweep over the time vector of the signal. Figure 16 plots the spectrograms with the three straight lines that represent the instantaneous frequencies imposed on it. Note that the narrow windows $(W \leq 64)$ are better in resolving the linear chirp than resolving the constant frequencies, while the wider windows $(W \geq 128)$ are better in resolving the constant frequencies.

However, notice that when applying the STFT the wider the window the less time resolution is obtained, the more end effect occurs and the more time truncation from both sides of the signal is taken. For example, Figure 16(f) only shows frequency information for the time period $12.8 \leq t \leq 17.2 \mathrm{~s}$ of the signal. Frequency information for periods $0 \leq t<12.8 \mathrm{~s}$ and $17.2<t \leq 30 \mathrm{~s}$ is not available, while the SOULTI wavelet spectrogram provides information for the duration of the signal as shown in Figure 14(b). Moreover, the SOULTI spectrogram resolves both the linear chirp and the constant harmonics, and its direct link between frequency (spectrograms) and scale (scalograms) allows checking the results for small or close frequencies.

\section{Edge effect mitigation}

Edge effect in harmonic and wavelet analysis of finite duration signals is caused by many factors. First, the measured signals are finite in duration and we do not have information about the signal after or before the times of recording. Second, many wavelets do not have compact support rather they have an effective window. Third, at the beginning of the analysis, $(\tau=0)$, the wavelet window is defined for negative and positive range of time, $t<0$ and $t>0$, but the analyzed signal is defined only for $t>0$, so the inner product is computed between the signal and part of the wavelet. Similarly, at the end of the analysis, the wavelet effective window will move out of the signal range and only part of it will take part in the inner product with the signal. This partial inner product gives inaccurate results at both edges.

The SOULTI wavelet is a right sided wavelet or signal, i.e. the mother wavelet is zero for $t<0$. Therefore, when performing the wavelet transform, the effective wavelet window sets fully inside the range of the signal at the beginning of the analysis when $\tau=0$. However, at the end of the analysis, the effective window moves out of the signal range and the inner product is performed between the signal and part of the effective window. Therefore, though the SOULTI wavelet solves naturally the edge effect at the beginning it does not solve the problem at the end, which makes the analysis at the end inaccurate and distorted.

As a solution for the end edge effect, we propose in this section performing a Reverse Wavelet Transform (RWT) analysis starting from the end of the signal. So the mother wavelet is reflected about $t=0$, then it is shifted to the end of the signal, and the wavelet analysis is performed end-to-start. Then, we reflect the results

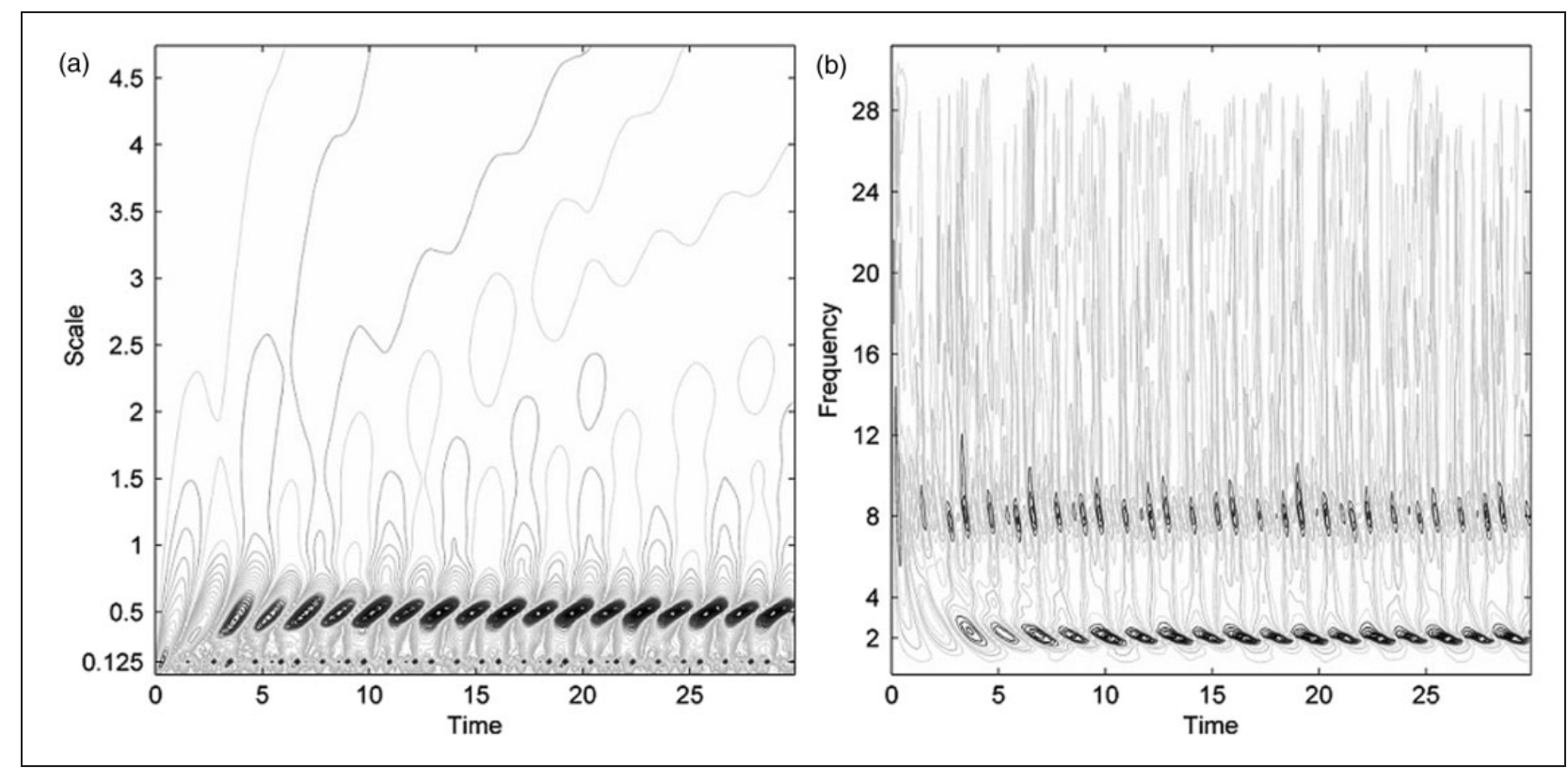

Figure 17. Reverse wavelet transform of the signal in Figure 10. (a) Scalogram, time-scale contour. (b) Spectrogram, time-frequency contour mapping. 


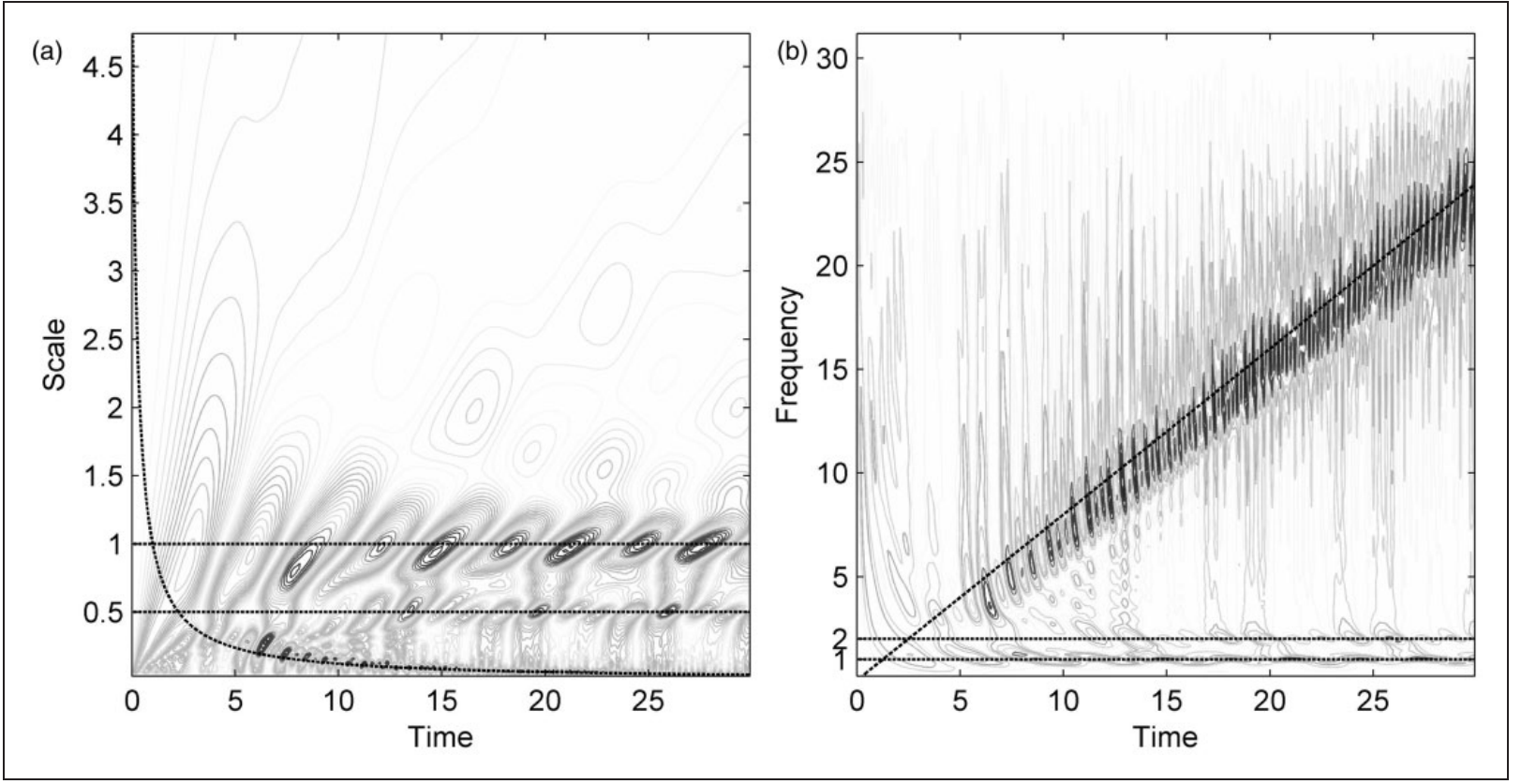

Figure 18. Reverse wavelet transform of the signal in Figure 12. (a) Scalogram, time-scale contour mapping. (b) Spectrogram, timefrequency contour mapping.
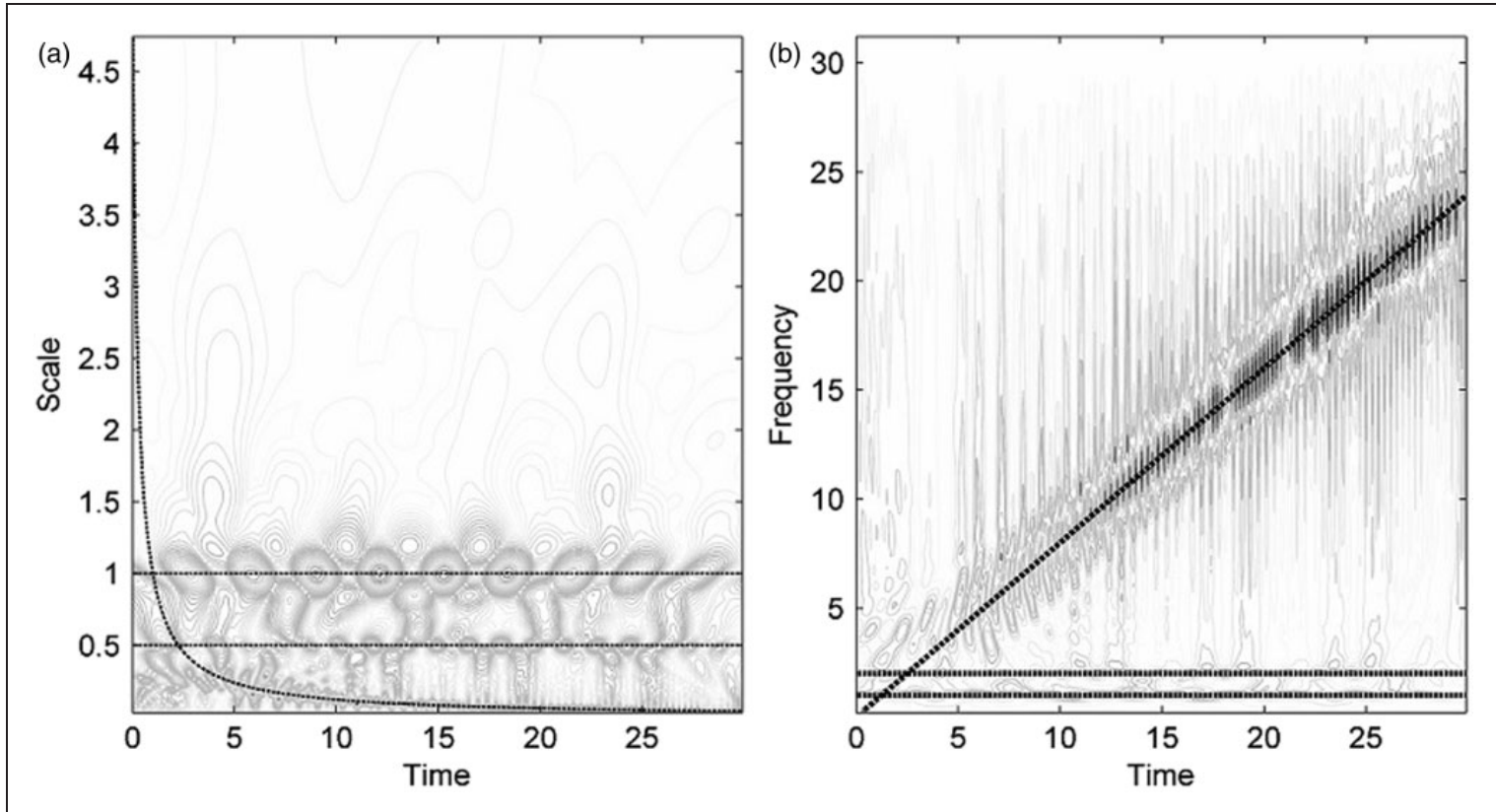

Figure 19. Average of FWT and RWT of the linear chirp in Figure 12. (a) Scalogram, time-scale contour mapping. (b) Spectrogram, time-frequency contour mapping.

back with respect to time. The same result can be obtained by just reflecting the signal, performing the wavelet analysis as usual and then reflecting the results back.

Figure 17 shows the RWT of the signal in Figure 10 and Figure 18 shows the RWT of the signal in
Figure 12. Note that at the end of the analysis there are clear ridges in both the scalograms and the spectrograms whereas the beginning shows distortions. This result gives an indication that the distortion of the ridges at the end of the studied signals is due to the edge effect. 
Instead of producing two scalograms or spectrograms for each signal, the average of the Forward Wavelet Transform (FWT) and the RWT can be computed and mapped to give refined results at both ends of the signal. Figure 19 shows the average of the FWT and RWT. The edges are better resolved and the distortion at the edges almost disappeared. However, a slight reduction in the ridges amplitude is notices.

\section{Remarks and conclusions}

It is shown that the impulse response of SOULTI systems can be used as a wavelet to obtain time-scale and time-frequency analysis directly. We also proved that the transform can be reversed to obtain the original signal, hence an inverse wavelet transform for the SOULTI wavelet exists. A region of convergence can be defined for the transform on the scale domain. This region defines in which range of scales the SOULTI wavelet transform converges.

Moreover, it is shown that the original signal can be retrieved back by substituting the transform into the conjugate differential equation. The SOULTI wavelet can be evaluated for most elementary functions and basic signals. In addition, there is a direct relation between the SOULTI wavelet transform of a signal and the transform of its derivative or integral. For a wavelet scaling power $p=1$, we found that the time-frequency resolution is preserved constant using the three definitions for computing the time-frequency resolution, the standard deviation based, the $-3 \mathrm{~dB}$ bandwidth based, and the Q-factor bandwidth based.

The important result that the reconstruction differential equation shows is extending the notion that the wavelet transform is the output of a filter bank from digital wavelets to crude noncompactly supported wavelets. The reconstruction differential equation in equation (19) shows that the SOULTI wavelet transform at scale $s$ is part of the output (particular solution) of the second-order system modeled by the differential equation itself.

The RWT can reveal whether the distortion at the end of the time range on scalograms and spectrograms is due to the edge effect or not. Moreover, taking the average between the FWT and RWT is a practical and simple method to eliminate the edge effect on both edges.

The SOULTI wavelet transform formula provides an analytical tool for time-frequency or time-scale representation of basic signals. It also preserves all the important characteristics and parameters that exist in the time domain to the time-scale or time-frequency domain.

\section{Declaration of Conflicting Interests}

The author(s) declared no potential conflicts of interest with respect to the research, authorship, and/or publication of this article.

\section{Funding}

The author(s) received no financial support for the research, authorship, and/or publication of this article.

\section{References}

Abuhamdia T and Taheri S (2015) Wavelets as a tool for systems analysis and control. Journal of Vibration and Control. Published online before print 16 December 2015. DOI: $10.1177 / 1077546315620923$.

Freudinger LC, Lind R and Brenner MJ (1998) Correlation filtering of modal dynamics using the Laplace wavelet. In: International modal analysis conference, volume 2, California (CA), Bethel, Connecticut (CT), USA, 2-5 February, pp.868-877. Santa Barbara, CA: SEM.

Hou Z and Hera A (2001) A system identification technique using pseudo-wavelets. Journal of Intelligent Material Systems and Structures 12(10): 681-687.

Jezequel L and Argoul P (1986) New integral transform for linear systems identification. Journal of Sound and Vibration 111(2): 261-278.

Kaiser G (1994) A Friendly Guide to Wavelets. Boston, MA: Birkhauser.

Meirovitch L (1997) Principles and Techniques of Vibrations. Vol. 1, Upper Saddle River, New Jersey: Prentice Hall.

Naylor AW and Sell GR (2000) Linear Operator Theory in Engineering and Science. New York: Springer Science \& Business Media.

Newland DE (1993) Harmonic wavelet analysis. Proceedings of the Royal Society of London. Series A: Mathematical and Physical Sciences 443(1917): 203-225.

Robinson EA (1962) Random Wavelets and Cybernetic Systems. Vol. 9, New York: Hafner Publishing Company.

Tchamitchan P and Torresani B (1992) Ridge and skeleton extraction from the wavelet transform. In: Ruskai MB, Gregory B and Ronald C (eds) Wavelets and Their Applications. Boston, MA: Jones and Bartlett Publishers, pp. $123-153$.

Yoshida K (1965) Functional Analysis. Vol. 123, 1st ed. Heidelberg: Springer-Verlag. 